\title{
Mandenkan
}

MANDENIKAN Bulletin semestriel d'études linguistiques mandé

$55 \mid 2016$

Numéro 55

\section{Textes tonalisés et glosés en soninké du Kingi}

Annexe 2

Denis Creissels

\section{(2) OpenEdition}

Journals

Édition électronique

URL : https://journals.openedition.org/mandenkan/739

DOI : 10.4000/mandenkan.739

ISSN : 2104-371X

Éditeur

Llacan UMR 8135 CNRS/Inalco

\section{Édition imprimée}

Date de publication : 30 juin 2016

Pagination : 131-172

ISSN : 0752-5443

\section{Référence électronique}

Denis Creissels, «Textes tonalisés et glosés en soninké du Kingi », Mandenkan [En ligne], 55 | 2016, mis en ligne le 01 juin 2016, consulté le 08 juillet 2021. URL : http://journals.openedition.org/mandenkan/ 739 ; DOI : https://doi.org/10.4000/mandenkan.739

Ce document a été généré automatiquement le 8 juillet 2021.

\section{(c) (i) (2)(2)}

Les contenus de Mandenkan sont mis à disposition selon les termes de la Licence Creative Commons Attribution - Pas d'Utilisation Commerciale - Partage dans les Mêmes Conditions 4.0 International. 


\section{Textes tonalisés et glosés en soninké du Kingi}

Annexe 2

Denis Creissels

\section{Contes}

\subsection{Conte 1 : l'âne de l'imam}

Ce texte, inspiré d'un conte traditionnel du Moyen-Orient, a été produit par Ismael Diawara dans le cadre du projet LGIDF (http://lgidf.cnrs.fr/)

Koota yogo, deben yalimaamin taaxallenman daga bug'a yi.

Un jour, un voisin de l'imam du village alla lui rendre visite.

\begin{tabular}{|l|l|l|l|l|l|}
\hline & Kòotá & yògó, & dèbé-n & yàlìmàamí-n & tàaxàllènmá-n \\
\hline & jour & un & village- $\mathrm{D}$ & imam- $\mathrm{D}^{\mathrm{BH}}$ & voisin- $\mathrm{D}^{\mathrm{BH}}$ \\
\hline
\end{tabular}

\begin{tabular}{|l|l|l|l|l|}
\hline & dàgá & búg(ú) & à & yí. \\
\hline & aller & sortir & $3 S G$ & POSTP \\
\hline
\end{tabular}

Taaxallenman ga kuuñindi, alimaamin d'a bisimilla.

Quand le voisin eut salué, l'imam l'accueillit.

\begin{tabular}{|l|l|l|l|l|l|l|l|}
\hline & Táaxállénmà-n & gà & kúuñîi-ndi, & àlimáamì-n & $\mathrm{d}(\mathrm{a})$ & à & bísímíllà. \\
\hline
\end{tabular}




\begin{tabular}{|l|l|l|l|l|l|l|l|}
\hline & voisin-D & SBD & saluer-ANTIP & imam.D & TR & 3SG & accueillir \\
\hline
\end{tabular}

Kuuñinden palle, taaxallenman da alimaamin tirindi, « An la w'an paren loxon'in ya ba?

Après les salutations, le voisin demanda à l'imam, «Est-ce que tu peux me prêter ton âne?

\begin{tabular}{|l|l|l|l|l|l|l|l|l|l|}
\hline & « Án & là & w(á) & án & pàrê-n & lóxó-n(ó) & ín & yà & bá ? \\
\hline & $2 S G$ & POT & INACP & $2 S G$ & âne-D ${ }^{\mathrm{BH}}$ & prêter-GER & $1 \mathrm{SG}$ & POSTP & $\mathrm{Q}$ \\
\hline
\end{tabular}

Yokku w'in maxa, n y'a munda n'i gana dagana debixooren di. »

J'ai des marchandises que je veux transporter à la ville. »

\begin{tabular}{|l|l|l|l|l|l|l|l|l|}
\hline & Yòkkú & w(á) & ín & màxá, & ń & y(á) & à & mùndá \\
\hline & marchandise.PL & COPLOC & $1 S G$ & SPHP & $1 S G$ & INACP & $3 S G$ & vouloir.GER \\
\hline
\end{tabular}

\begin{tabular}{|l|l|l|l|l|l|l|}
\hline & $\mathbf{n}(\grave{a})$ & $\mathbf{i}$ & gàná & dàgà-ná & dèbì-xòorén & dì. » \\
\hline & INF & $3 \mathrm{PL}$ & transporter & aller-GER & village-grand-D & dans \\
\hline
\end{tabular}

Alimaamin ma ñ'a munda n'i haren lox'a yi.

L'imam n'avait pas envie de lui prêter son âne.

\begin{tabular}{|l|l|l|l|l|l|l|}
\hline & Àlimáamì-n & má & $\tilde{\mathbf{n}}(\mathbf{i})$ & $\mathbf{a ̀}$ & mùndá & \\
\hline & imam-D & NEG & PAS $^{1}$ & $3 S G$ & vouloir.GER & \\
\hline
\end{tabular}

\begin{tabular}{|l|l|l|l|l|l|l|}
\hline & $\mathbf{n}(\mathbf{a})$ & $\mathbf{i}$ & hàrê-n & lóx(ó) & à & yí. \\
\hline & INF & REFL & âne-D & prêter & $3 S G$ & POSTP \\
\hline
\end{tabular}

A ga ma ñ'a munda na taaxallenman ñaagundi, a d'a jaabi, «Haren ga ñi yere, $n$ ñ'a loxon'an ya, q'in d'a loxo sere tana yi. "

Comme il ne voulait pas vexer le voisin, il répondit, «Si l'âne était ici, je te le prêterais, mais je l'ai prêté à une autre personne. »

\begin{tabular}{|l|l|l|l|l|l|l|}
\hline & À & gà & má & $\tilde{\mathbf{n}}(\mathbf{i})$ & à & mùndá \\
\hline & $3 S G$ & $\mathrm{SBD}$ & $\mathrm{ACP}$ & $\mathrm{PAS}$ & $3 \mathrm{SG}$ & vouloir \\
\hline
\end{tabular}




\begin{tabular}{|l|l|l|l|l|l|l|l|}
\hline & nà & táaxállénmà-n & ñàagù-ndí, & à & $\mathbf{d}(\mathbf{a})$ & à & jáabì, \\
\hline & INF & voisin.D & avoir_honte-CAUS & $3 S G$ & TR & $3 S G$ & répondre \\
\hline
\end{tabular}

\begin{tabular}{|c|c|c|c|}
\hline « Hàré-n & gà & ñ́í & yèré, \\
\hline âne-D & SBD & se_trouver & ici \\
\hline
\end{tabular}

\begin{tabular}{|l|l|l|l|l|l|l|}
\hline & ń & $\tilde{\mathbf{n}}(\mathbf{i})$ & à & lóxó-n(ó) & án & yà, \\
\hline & $1 S G$ & PAS & $3 S G$ & prêter-GER & $2 S G$ & POSTP \\
\hline
\end{tabular}

\begin{tabular}{|l|l|l|l|l|l|l|l|l|}
\hline & q(à) & în & $\mathbf{d}(\mathbf{a})$ & à & lóxó & sèré & tàná & yì. » \\
\hline & mais & $1 S G$ & TR & $3 S G$ & prêter & personne & autre & POSTP \\
\hline
\end{tabular}

Ken d'a ñi, haren ciitinten ya halle, a qusa nan qaaru.

Il se trouva que l'âne, qui était attaché derrière [la maison], se mit à braire.

\begin{tabular}{|l|l|l|l|l|l|l|l|l|}
\hline & Kén & $\mathbf{d}(\mathbf{a})$ & $\mathbf{a}$ & ñí, & hàré-n & cìitì-ntê-n & yá & hállè ; \\
\hline & DEM & TR & $3 S G$ & se_trouver & âne-D & attacher-RES-D ${ }^{\mathrm{BH}}$ & être & derrière \\
\hline
\end{tabular}

\begin{tabular}{|l|l|l|l|l|}
\hline & à & qúsà & nàn & qáarù. \\
\hline & $3 S G$ & se_mettre_à & INF & braire \\
\hline
\end{tabular}

Taaxallenman godo, « An ti mani ? N ñinme, n taron ya haren qaaruxannen ya kan palle!»

Le voisin s'exclama, « Qu'est-ce que tu dis ? Moi-même, j'entends l'âne braire derrière la maison. »

\begin{tabular}{|l|l|l|l|l|l|}
\hline & Táaxállénmà-n & gódò, & « Án & tì & màní ? \\
\hline & voisin-D & s'exclamer & $2 S G$ & dire & quoi \\
\hline
\end{tabular}

\begin{tabular}{|l|l|l|l|l|l|l|}
\hline & Ń & ñìnmé, & ń & tàrô-n & yá & \\
\hline & $1 S G$ & INT $^{2}$ & $1 S G$ & oreille-D & COPLOC & \\
\hline
\end{tabular}

\begin{tabular}{|l|l|l|l|l|l|}
\hline & hàré-n & qàarù-xànné-n & yà & kán & pàllé ! \\
\hline & âne-D & crier-voix- $\mathrm{D}^{\mathrm{BH}}$ & POSTP & maison.D & derrière \\
\hline
\end{tabular}


Alimaamin qa butunten godo, « An nta yaagunu?

Et l'imam répliqua fâché, « Est-ce que tu n'as pas honte?

\begin{tabular}{|l|l|l|l|l|}
\hline & Àlimáamì-n & qá & bùtù-ntê-n & gódò, \\
\hline & imam-D & aussi & se_fâcher-RES-D & \\
\hline
\end{tabular}

\begin{tabular}{|l|l|l|l|l|}
\hline & « Án & ntá & yàagù-nù ? \\
\hline & $2 S G$ & INACP.NEG & avoir_honte.GER & \\
\hline
\end{tabular}

An caqqa haren ña diganqannen ya diina alimaamin ya ba?

Est-ce que c'est à la parole de l'âne que tu fais confiance plutôt qu'à [celle de] l'imam?»

\begin{tabular}{|l|l|l|l|l|l|l|}
\hline & Án & cáqqá & hàré-n & ñà & digàn-qànnén & yà \\
\hline & $2 S G$ & faire_confiance.GER & âne-D & FOC & parole-voix-D $\mathrm{D}^{\mathrm{BH}}$ & POSTP \\
\hline
\end{tabular}

\begin{tabular}{|l|l|l|l|l|}
\hline & díinà & àlimáamì-n & yá & bà ? » \\
\hline & plus_que & imam-D & POSTP & $\mathrm{Q}$ \\
\hline
\end{tabular}

\subsection{Conte 2 : Les trois amis}

Plusieurs versions de ce conte soninké ont déjà été publiées, notamment par Doucouré (2015) qui confronte sa propre version avec les précédentes dans la perspective d'une discussion des choix orthographiques. Celle-ci est la transcription d'une reformulation effectuée oralement par Ismael Diawara.

Ku yugu sikki ñi menjanyun ña yi.

C'est l'histoire de trois amis.

\begin{tabular}{|l|l|l|l|l|l|l|l|}
\hline & Kú & yúgú & sìkkì & $\tilde{\mathbf{n i}}$ & mènjányù-n & $\tilde{\text { ná }}$ & ỳ̀. \\
\hline & DEM.PL & homme.PL & TROIS & COPEQ & ami.PL-D & FOC & POSTP \\
\hline
\end{tabular}

Baanen paaba d'a qata,

Le premier a été chassé par son père

\begin{tabular}{|l|l|l|l|l|l|}
\hline & Báanè-n & pàabá & $d(a ̀)$ & à & qátà, \\
\hline
\end{tabular}




\begin{tabular}{|l|l|l|l|l|l|}
\hline & un-D & père $^{\mathrm{BH}}$ & $\mathrm{TR}$ & $3 \mathrm{SG}$ & chasser \\
\hline
\end{tabular}

nan t'a wa yaxarinkutini moxoburu.

parce qu'il court trop après les femmes.

\begin{tabular}{|l|l|l|l|l|l|l|}
\hline & nàn & $\mathbf{t}(\mathbf{i})$ & à & wá & yàxàrì-n-kútí-ní & móxó-búrù. \\
\hline & INF & dire & $3 S G$ & INACP & femme-EP-couper.DETR-GER & manière-mauvais \\
\hline
\end{tabular}

Baanen paaba d'a qata,

Le deuxième $a$ été chassé par son père

\begin{tabular}{|c|c|c|c|c|}
\hline Báanè-n & pàabá & $d(a)$ & à & qátà, \\
\hline un-D & père ${ }^{B H}$ & TR & $3 S G$ & chasser \\
\hline
\end{tabular}

nan t'a wa tigakanbini moxoburu.

parce qu'il croque trop d'arachide

\begin{tabular}{|l|l|l|l|l|l|l|}
\hline & nàn & $\mathbf{t}(\mathbf{i})$ & $\mathbf{a ̀}$ & wá & tìgà-kànbì-ní & móxó-búrù. \\
\hline & INF & dire & $3 S G$ & INACP & arachide-croquer.DETR-GER & manière-mauvais \\
\hline
\end{tabular}

Baanen paaba d'a qata,

Le troisième a été chassé par son père

\begin{tabular}{|l|l|l|l|l|l|}
\hline & Báanè-n & pàabá & $\mathbf{d}(\mathbf{a})$ & à & qátà, \\
\hline & un-D & père $^{\mathrm{BH}}$ & $\mathrm{TR}$ & $3 \mathrm{SG}$ & chasser \\
\hline
\end{tabular}

nan t'a wa suuriyigene moxoburu.

parce qu'il mange trop de to.

\begin{tabular}{|l|l|l|l|l|l|l|}
\hline & nàn & $\mathbf{t}(\mathbf{i})$ & $\mathbf{a}$ & wá & sùurì-yígé-né & móxó-búrù. \\
\hline & INF & dire & $3 S G$ & INACP & to-manger.DETR-GER & manière-mauvais \\
\hline
\end{tabular}

I da killen kahu nan daga yanqa tunka ya.

Ils sont partis ensemble et sont allés loger chez un roi. 


\begin{tabular}{|l|l|l|l|l|l|l|l|l|l|}
\hline & I & dà & kìllé-n & kàhú & nàn & dàgá & yànqá & tùnká & yà. \\
\hline & $3 \mathrm{PL}$ & $\mathrm{TR}$ & route-D & réunir & $\mathrm{INF}$ & aller & descendre & roi & FOC \\
\hline
\end{tabular}

Suuriyigaana, a da kame suurinñokke kin'a yi,

Celui-ci a donné au mangeur de to cent marmites de to,

\begin{tabular}{|l|l|l|l|l|l|l|l|l|}
\hline & Sùurì-yíg-áanà, & à & dà & kàmé & sùurì-n-ñòkké & kín(i) & à & yí, \\
\hline & to-manger-NMAG.D & $3 S G$ & TR & cent & to-EP-récipient ${ }^{\mathrm{BH}}$ & donner & $3 \mathrm{SG}$ & POSTP \\
\hline
\end{tabular}

tigakanbaana, a da kame tigakande kin'a yi,

il a donné au croqueur d'arachide cent paniers d'arachide,

\begin{tabular}{|l|l|l|l|l|l|}
\hline & tìgà-kànb-áanà, & à & dà & kàmé & tìgà-kàndé \\
\hline & arachide-croquer-NMAG.D & $3 \mathrm{SG}$ & $\mathrm{TR}$ & cent & arachide-panier $^{\mathrm{BH}}$ \\
\hline
\end{tabular}

\begin{tabular}{|l|l|l|l|}
\hline & kín(i) & à & yí, \\
\hline & donner & $3 S G$ & POSTP \\
\hline
\end{tabular}

qusukutaana, a da kame quso kin'a yi.

et il a donné au coureur de jupons cent jeunes filles.

\begin{tabular}{|l|l|l|l|l|l|l|l|l|}
\hline & qùsù-kút-áanà, & à & dà & kàmé & qùsó & kín(i) & à & yí. \\
\hline & fille-couper-NMAG.D & $3 S G$ & TR & cent & fille ${ }^{\mathrm{BH}}$ & donner & $3 S \mathrm{SG}$ & POSTP \\
\hline
\end{tabular}

Wuron ga ro, a da baane su rondi konpen di

Quand il a fait nuit, il a fait entrer chacun d'entre eux dans une pièce

\begin{tabular}{|l|l|l|l|l|l|l|l|l|l|l|}
\hline & Wùró-n & gà & ró, & à & dà & báané & sú & ró-ndí & kónpè-n & dí \\
\hline & nuit-D & SBD & entrer & $3 S G$ & $\mathrm{TR}$ & un & tous & entrer-CAUS & chambre-D & D \\
\hline
\end{tabular}

n'a raqqen tex'a yi.

et il les a enfermés

\begin{tabular}{|l|l|l|l|l|l|l|}
\hline & n(à) & á & ràqqêên & téx(é) & à & yí. \\
\hline
\end{tabular}




\begin{tabular}{|c|c|c|c|c|c|}
\hline INF & $3 \mathrm{SG}^{\mathrm{H}}$ & porte- $\mathrm{D}^{\mathrm{BH}}$ & fermer & $3 S G$ & POSTP \\
\hline
\end{tabular}

Yillen ga karene,

Quand le jour s'est levé,

\begin{tabular}{|l|l|l|l|}
\hline & Yíllè-n & gá & káré-né, \\
\hline & cycle-D & SBD.INACP & se-casser-GER \\
\hline
\end{tabular}

i daga katti tigakanbaanan ya,

les gens sont allés voir le croqueur d'arachide,

\begin{tabular}{|l|l|l|l|l|l|}
\hline & ì & dàgá & káttì & tìgà-kànb-áanà-n & yá, \\
\hline & $3 P L$ & aller & vers & arachide-croquer-NMAG-D & POSTP \\
\hline
\end{tabular}

i d'a ñi a wa wuunu,

et ils l'ont trouvé en train de pleurer

\begin{tabular}{|l|l|l|l|l|l|l|l|}
\hline & $\mathbf{i}$ & $\mathbf{d}(\mathbf{a})$ & $\mathbf{a ̀}$ & $\tilde{\mathbf{n i}} \mathbf{i}$ & $\mathbf{a ̀}$ & wá & wúu-nú, \\
\hline & $3 P L$ & TR & $3 S G$ & trouver & $3 S G$ & INACP & pleurer-GER \\
\hline
\end{tabular}

na tigakoromun jaga,

et de fouiller dans les coques d'arachide,

\begin{tabular}{|l|l|l|l|}
\hline & nà & tìgà-kórómù-n & jàgá, \\
\hline & INF & arachide-coque.PL-D & creuser \\
\hline
\end{tabular}

t'i d'ike tigan cu kanbu.

parce qu'il avait mangé toutes ses arachides.

\begin{tabular}{|l|l|l|l|l|l|l|l|}
\hline & $\mathbf{t}(\mathbf{i})$ & $\hat{\mathbf{i}}$ & $\mathbf{d}(\mathbf{a})$ & í-ké & tìgâ-n & cú & kànbú. \\
\hline & que & REFL & TR & REFL-EMPH & arachide-D ${ }^{\mathrm{BH}}$ & tout & croquer \\
\hline
\end{tabular}

I dangi nan li suuriyigaanan ya,

Ils sont passés au mangeur de to, 


\begin{tabular}{|l|l|l|l|l|l|l|}
\hline & İ & dángì & nàn & lí & sùurì-yíg-áanà-n & yá, \\
\hline & $3 \mathrm{PL}$ & passer & INF & venir & to-manger-NMAG-D & POSTP \\
\hline
\end{tabular}

i d'a ñi ken qa wa noorun koosini,

et ils l'ont trouvé en train de racler les écuelles,

\begin{tabular}{|l|l|l|l|l|l|l|l|l|l|}
\hline & ì & $\mathbf{d}(\mathbf{a})$ & $\mathbf{a}$ & $\tilde{\mathbf{n i}}$ & kên & qá & wá & nóorù-n & kòosì-ní, \\
\hline & $3 \mathrm{PL}$ & $\mathrm{TR}$ & $3 \mathrm{SG}$ & trouver & DEM & aussi & INACP & écuelle.PL-D & racler-GER \\
\hline
\end{tabular}

t’i d'i suuren cu yiga.

parce qu'il avait mangé tout son to.

\begin{tabular}{|l|l|l|l|l|l|l|l|}
\hline & $\mathbf{t}(\mathbf{i})$ & $\hat{\mathbf{i}}$ & $\mathbf{d}(\mathbf{a})$ & $\mathbf{i}$ & sùurê-n & cú & yígá. \\
\hline & que & REFL & TR & REFL & to-D $\mathrm{D}^{\mathrm{BH}}$ & tout & manger \\
\hline
\end{tabular}

I dangi nan li qusukutaanan ya.

Ils sont passés au coureur de jupons.

\begin{tabular}{|l|l|l|l|l|l|l|}
\hline & I & dángì & nàn & lí & qùsù-kút-áanà-n & yá. \\
\hline & $3 P L$ & passer & INF & venir & fille-couper-NMAG-D & POSTP \\
\hline
\end{tabular}

I ga t'i wa laqqen yuñini,

Au moment où ils allaient ouvrir la porte,

\begin{tabular}{|l|l|l|l|l|l|l|l|}
\hline & Ì & gà & $\mathbf{t}(\mathbf{i})$ & ì & wá & láqqè-n & yùñì-ní, \\
\hline & 3 PL & SBD & dire & 3PL & INACP & porte-D & ouvrir-GER \\
\hline
\end{tabular}

a renñugusooman t'i n'maxa laqqen yuñi,

son fils aîné leur a dit de ne pas ouvrir la porte,

\begin{tabular}{|c|c|c|c|c|c|c|c|}
\hline á & rèn-ñùgù-sòomá-n & $\mathbf{t}(\mathbf{i})$ & ì & n(àn) & máxà & láqqè-n & yùñí, \\
\hline $3 \mathrm{SG}^{\mathrm{H}}$ & enfant-mâle-aîné-D $\mathrm{D}^{\mathrm{BH}}$ & dire & 3PL & SUBJ & $\mathrm{PROH}$ & porte-D & ouvrir \\
\hline
\end{tabular}

i haaba ma giri qenqon di. 
car son père n'est pas encore levé.

\begin{tabular}{|l|l|l|l|l|l|l|}
\hline & î̀ & hàabá & má & gìrì & qènqó-n & di. \\
\hline & REFL & père & ACP.NEG & se_lever & sommeil-D & dans \\
\hline
\end{tabular}

Kan qa n'qoto ku di?

Parmi eux, lequel est donc le plus fort?

\begin{tabular}{|l|l|l|l|l|l|l|}
\hline & Kân & qá & (nà)n & qòtò & kú & dì? \\
\hline & lequel ? & aussi & ST & être_fort & DEM.PL & dans \\
\hline
\end{tabular}

\subsection{Conte 3 : Si on laisse une femme décider du sort de sa co- épouse, elle la tuera}

Ce conte ainsi que les deux suivants font partie des contes qui développent ou expliquent un proverbe. Des versions de ces trois contes en soninké du Gidinmaxa figurent dans Tunkara et Bonmu (2012) ${ }^{4}$. Les versions données ici sont la transcription d'une reformulation effectuée oralement par Ismael Diawara.

Ke ña yaxare yogo ya yi, a ga daga i tongen linjondi tagayugon banyen ya kandaaran ya.

C'est l'histoire d'une femme qui était allée faire aiguiser sa houe chez un forgeron à l'approche de l'hivernage.

\begin{tabular}{|l|l|l|l|l|l|l|}
\hline & Ké & ñá & yàxàré & yògó & yà & yí, \\
\hline & DEM & être $^{5}$ & femme & INDEF & FOC & POSTP \\
\hline
\end{tabular}

\begin{tabular}{|l|l|l|l|l|l|l|}
\hline & à & gà & dàgá & î̀ & tòngê-n & línyó-ndí \\
\hline & $3 S G$ & $S B D$ & aller & REFL & houe- $\mathrm{D}^{\mathrm{BH}}$ & être_bon-CAUS \\
\hline
\end{tabular}

\begin{tabular}{|l|l|l|l|l|l|}
\hline & tàgà-yúgò-n & bànyé-n & yà & kàndáarà-n & yá. \\
\hline & forgeron-homme & côté-D $\mathrm{D}^{\mathrm{BH}}$ & POSTP & temps_avant_l'hivernage-D & POSTP \\
\hline
\end{tabular}

Tage ke d'a ro yinben ya, a ga d'a bugu yinben ya, n'a linjondi tanni, yaxaren qusa ri katta tagen ya, t'i r'i tongen yutu.

Le forgeron en question la mit au feu, et dès qu'il l'eut sortie du feu et aiguisée, la femme vint chez le forgeron, dans l'intention de prendre sa houe. 


\begin{tabular}{|l|l|l|l|l|l|l|l|}
\hline & Tàgé & ké & $\mathbf{d}(\mathbf{a})$ & à & ró & yìnbé-n & yà, \\
\hline & forgeron & DEM & TR & $3 S G$ & mettre & feu-D & POSTP \\
\hline
\end{tabular}

\begin{tabular}{|l|l|l|l|l|l|l|l|}
\hline & $\mathbf{a}$ & gà & $\mathbf{d}(\mathbf{a})$ & à & búgú & yìnbé-n & yà, \\
\hline & $3 S G$ & SBD & TR & $3 S G$ & sortir & feu-D & POSTP \\
\hline
\end{tabular}

\begin{tabular}{|l|l|l|l|l|}
\hline & $\mathbf{n}(\mathbf{a})$ & $\mathbf{a}$ & línyóndí & tánní, \\
\hline & INF & $3 S G$ & être_bon_CAUS & seulement \\
\hline
\end{tabular}

\begin{tabular}{|l|l|l|l|l|l|l|}
\hline & yàxàrê-n & qúsà & rí & káttà & tàgé-n & yà, \\
\hline & femme-D & faire_aussitôt & venir & vers & forgeron-D & POSTP \\
\hline
\end{tabular}

\begin{tabular}{|l|l|l|l|l|l|l|}
\hline & $\mathbf{t}(\mathbf{i})$ & $\hat{\mathbf{i}}$ & $\mathbf{r ( i )}$ & $\hat{\mathbf{i}}$ & tòngé-n & yùtú. \\
\hline & que $^{6}$ & REFL & venir & REFL & houe-D $^{\mathrm{BH}}$ & prendre \\
\hline
\end{tabular}

Tagen $t i$ « An tongen payi, qa max'a ro an kunken ya de, ken ga he, an tayinnen ya kalla ».

Le forgeron dit, «Voici ta houe, mais ne la mets pas à l'épaule, sinon ta coépouse mourra. »

\begin{tabular}{|l|l|l|l|l|l|}
\hline & Tàgé-n & tì, & « án & tòngê-n & páyí, \\
\hline & forgeron-D & dire & $2 S G$ & houe- $\mathrm{D}^{\mathrm{BH}}$ & regarder \\
\hline
\end{tabular}

\begin{tabular}{|l|l|l|l|l|l|l|l|l|}
\hline & qà & máx(à) & à & ró & án & kùnké-n & yà & dé, \\
\hline & mais & PROH & $3 S G$ & mettre & $2 S G$ & épaule-D ${ }^{\mathrm{BH}}$ & POSTP & EMPH \\
\hline
\end{tabular}

\begin{tabular}{|c|c|c|c|c|c|c|}
\hline kén & gà & hé, & án & tàyìnnê-n & yá & kàllá. " \\
\hline DEM & SBD & COPEQ.NEG & $2 S G$ & co_épouse- $\mathrm{D}^{\mathrm{BH}}$ & INACP & mourir.GER \\
\hline
\end{tabular}

Yaxaren ga da tongen yutu, a ga bugu a d'i kaaron payi, a ga ma sere yari, a ti, «Jaaxa ke ya ga ñaana tayinnen caaten ya, a wa kalla saasa. ”

La femme prit la houe, sortit et regarda autour d'elle, et ne voyant personne, elle dit «Donc si c'est à ça que tient le sort de ma co-épouse, elle va mourir tout de suite.» 


\begin{tabular}{|l|l|l|l|l|l|}
\hline & Yàxàré-n & gà & dà & tóngè-n & yùtú, \\
\hline & femme-D & SBD & TR & houe-D & prendre \\
\hline
\end{tabular}

\begin{tabular}{|l|l|l|l|l|l|l|l|l|}
\hline & à & gà & búgú & à & $\mathbf{d}(\mathbf{a})$ & í & kàarô-n & páyí, \\
\hline & $3 S G$ & SBD & sortir & $3 S G$ & TR & REFL & côté.PL-D & regarder \\
\hline
\end{tabular}

\begin{tabular}{|l|l|l|l|l|l|l|l|}
\hline & à & gà & má & sèré & yàrì, & à & tí \\
\hline & $3 S G$ & SBD & ACP.NEG & personne & voir $^{B}$ & $3 S G$ & dire \\
\hline
\end{tabular}

\begin{tabular}{|l|l|l|l|l|l|}
\hline & « Jáaxà & ké & yà & gá & ñàa-nà \\
\hline & donc & DEM & FOC & SBD.INACP & être-GER \\
\hline
\end{tabular}

\begin{tabular}{|l|l|l|l|l|l|l|l|}
\hline & tàyìnné-n & càaté-n & yà, & à & wá & kàllá & sáasá. » \\
\hline & co_épouse-D & destin- $\mathrm{D}^{\mathrm{BH}}$ & POSTP & $3 \mathrm{SG}$ & INACP & mourir.GER & maintenant \\
\hline
\end{tabular}

A qusa tonge ke ro i kunken ya, tongen d'a kunken bi.

Elle mit aussitôt la houe en question à son épaule, et la houe lui brûla l'épaule.

\begin{tabular}{|l|l|l|l|l|l|l|l|l|}
\hline & À & qúsà & tóngè & ké & ró & ì & kùnké-n & yà, \\
\hline & $3 S G$ & faire_aussitôt & houe & DEM & mettre & REFL & épaule-D ${ }^{\mathrm{BH}}$ & POSTP \\
\hline
\end{tabular}

\begin{tabular}{|l|l|l|l|l|l|}
\hline & tóngè-n & $\mathbf{d}(\mathbf{a})$ & á & kùnkê-n & bíyí. \\
\hline & houe-D & $\mathrm{TR}$ & $3 \mathrm{SG}$ & épaule- $\mathrm{D}^{\mathrm{BH}}$ & brûler \\
\hline
\end{tabular}

A qus'a harati bakka kunken ya.

Elle l'arracha aussitôt de son épaule.

\begin{tabular}{|l|l|l|l|l|l|l|l|}
\hline & À & qús(à) & à & hàràtí & bákká & kúnkè-n & yá. \\
\hline & $3 S G$ & faire_aussitôt & $3 S G$ & arracher & sortir-GER & épaule-D & POSTP \\
\hline
\end{tabular}

A ga daga kan ya, i ga d'a tirindi, ti mani qan d'a kita i kunken ya, a ma dunje na hooho ko.

A son retour à la maison, on lui demanda ce qui lui était arrivé à l'épaule, mais elle refusa de dire quoi que ce soit. 


\begin{tabular}{|l|l|l|l|l|l|l|l|l|l|l|}
\hline & À & gà & dàgá & ká-n & yà, & ì & gà & d(à) & à & tírìndí, \\
\hline & $3 S G$ & SBD & aller & maison & POSTP & $3 \mathrm{PL}$ & $\mathrm{SBD}$ & $\mathrm{TR}$ & $3 \mathrm{SG}$ & interroger \\
\hline
\end{tabular}

\begin{tabular}{|l|l|l|l|l|l|l|l|l|l|}
\hline & tí & màní & qá-n & $\mathbf{d}(\mathbf{a})$ & $\mathbf{a}$ & kìtà & $\mathbf{i}$ & kùnké-n & yà, \\
\hline & que & quoi & aussi-S & TR & $3 S G$ & affecter $^{B}$ & REFL & épaule-D $^{\mathrm{BH}}$ & POSTP \\
\hline
\end{tabular}

\begin{tabular}{|l|l|l|l|l|l|l|}
\hline & à & má & dùnyè & nà & hóohò & kó. \\
\hline & $3 S G$ & ACP.NEG & accepter & & & \\
\hline H & INF & quoi_que_ce_soit & dire \\
\hline
\end{tabular}

Ken ñan d'a koyi, t'an ga na tayinnen caaten kini a meetayinnen ya, a w'a karini.

C'est ce qui montre que si on confie le sort de l'épouse d'un mari polygame à sa co-épouse, elle la tuera.

\begin{tabular}{|l|l|l|l|l|l|}
\hline & Kén & ñà-n & $\mathbf{d}(\mathbf{a})$ & $\mathbf{a ̀}$ & kòỳ̀, \\
\hline & DEM & FOC-S & TR & $3 S G$ & montrer \\
\hline
\end{tabular}

\begin{tabular}{|l|l|l|l|l|l|l|l|}
\hline & $\mathbf{t}(\mathbf{i})$ & án & gà & ná & tàyìnné-n & càaté-n & kìnì \\
\hline & que & $2 \mathrm{SG}$ & $\mathrm{SBD}$ & $\mathrm{PROJ}$ & co_épouse-D & destin- $\mathrm{D}^{\mathrm{BH}}$ & donner $^{\mathrm{B}}$ \\
\hline
\end{tabular}

\begin{tabular}{|l|l|l|l|l|l|l|l|}
\hline & á & mèe-tàyìnné-n & yà, & à & w(á) & à & kàrì-ní. \\
\hline & $3 S G^{\mathrm{H}}$ & RECIP-Co_épouse- ${ }^{\mathrm{BH}}$ & POSTP & $3 S \mathrm{SG}$ & INACP & $3 S \mathrm{SG}$ & tuer-GER \\
\hline
\end{tabular}

\subsection{Conte 4 : On peut dire de quelqu'un « C'est mon ancien ami, » mais pas « C'est mon ancien frère. »}

I ga ti «Qanasiren nan pasu waaxiburen ya, » ken ni gaaren ña yi, bawo seren yaaxin ga na bono moxo wo moxo, an la wa tini « Kaati n'in qanaxasen ya, ", qa " Kaati n'in yaaxixasen ya, » ken ni gaaren ña yi.

Quand on dit qu'un bon ami vaut mieux qu'un mauvais frère, c'est faux, car aussi mauvais que puisse être un frère, on peut dire «Untel est mon ancien ami, » mais « Untel est mon ancien frère, » c'est faux.

\begin{tabular}{|l|l|l|l|l|l|l|}
\hline & I & gà & tî & « Qànà-sírè-n & nàn & pàsú \\
\hline & 3PL & SBD & dire & ami-bon-D & ST & valoir_mieux \\
\hline
\end{tabular}




\begin{tabular}{|l|l|l|l|l|l|l|l|}
\hline & wáaxí-búrè-n & yá, » & kén & nì & gàaré-n & ñà & yí, \\
\hline & frère-mauvais-D & POSTP & DEM & COPEQ & mensonge-D & FOC & POSTP \\
\hline
\end{tabular}

\begin{tabular}{|l|l|l|l|l|l|l|}
\hline & báwò & sèré-n & yàaxí-n & gà & ná & bònò \\
\hline & parce_que & personne-D & frère-D $^{\mathrm{BH}}$ & $\mathrm{SBD}$ & $\mathrm{PROJ}$ & s'abîmer \\
\hline
\end{tabular}

\begin{tabular}{|l|l|l|l|l|l|l|l|}
\hline & mòxó & wó & mòxò, & án & là & wá & tì-ní \\
\hline & manière & DISTR & manière $^{\mathrm{B}}$ & $2 \mathrm{SG}$ & $\mathrm{POT}$ & $\mathrm{INACP}$ & dire-GER \\
\hline
\end{tabular}

\begin{tabular}{|l|l|l|l|l|l|}
\hline & « Kàatí & $\mathbf{n}(\mathbf{i})$ & ín & qànà-xàsé-n & yà, " \\
\hline & Untel & COPEQ & $1 S G$ & ami-ancien-D & \\
\hline
\end{tabular}

\begin{tabular}{|c|c|c|c|c|c|}
\hline qà & « Kàatí & n(i) & în & yàaxì-xàsé-n & yà, " \\
\hline mais & Untel & COPEQ & $1 S G$ & frère-ancien- $\mathrm{D}^{\mathrm{BH}}$ & POSTP \\
\hline
\end{tabular}

\begin{tabular}{|l|l|l|l|l|l|}
\hline & kén & nì & gàaré-n & ñà & yí. \\
\hline & DEM & COPEQ & mensonge-D & FOC & POSTP \\
\hline
\end{tabular}

Ho be me ga ntaxa kitene badabada, ho wa sirono na ken baga ba?

Une chose dont on ne retrouvera jamais l'équivalent, qu'est-ce qui peut l'égaler?

\begin{tabular}{|l|l|l|l|l|l|l|l|}
\hline & Hó & bè & mé & gà & ntáxà & kìtè-né & bádábádá, \\
\hline & chose & REL & équivalent & SBD & CESS & obtenir.DETR-GER & jamais \\
\hline
\end{tabular}

\begin{tabular}{|l|l|l|l|l|l|l|l|}
\hline & hó & wá & síró-nó & nà & kên & bágá & bà ? \\
\hline & chose & INACP & devenir_bon-GER & INF & DEM & égaler & Q \\
\hline
\end{tabular}

Qanaaxun la na sirono a ga qotono maarenmaaxun ya kan moxo? Misaale :

Comment l'amitié pourrait-elle être meilleure et plus forte que la fraternité ? Par exemple :

\begin{tabular}{|l|l|l|l|l|l|l|}
\hline & Qànàaxú-n & là & nà & síró-nó & nàn & qótò-nó \\
\hline
\end{tabular}




\begin{tabular}{|l|l|l|l|l|l|l|}
\hline & amitié-D & POT & INACP & devenir_bon-GER & INF & devenir_dur \\
\hline
\end{tabular}

\begin{tabular}{|l|l|l|l|l|l|}
\hline & máarènmàaxú-n & yà & kán & mòxó ? & Mìsáalè : \\
\hline & fraternité-D & POSTP & quel ? & manière & exemple \\
\hline
\end{tabular}

Yugo yogo ya ni, a d'i waaxin ya me maxa kan ya, i su haababaane, q'i nta saaxe yi.

C'est un homme qui vit dans la même maison que son frère, ils sont de même père mais de mères différentes.

\begin{tabular}{|l|l|l|l|l|}
\hline & Yúgó & yògó & yà & ní, \\
\hline & homme & INDEF & FOC & COPEQ \\
\hline
\end{tabular}

\begin{tabular}{|l|l|l|l|l|l|l|l|l|l|}
\hline & á & $\mathbf{d}(\grave{o})$ & $\hat{\mathbf{i}}$ & wàaxí-n & yà & mé & màxá & ká-n & yà, \\
\hline & $3 S G^{\mathrm{H}}$ & avec & REFL & frère- $\mathrm{D}^{\mathrm{BH}}$ & POSTP & RECIP & SPHP & maison-D & POSTP \\
\hline
\end{tabular}

\begin{tabular}{|l|l|l|l|l|l|l|l|l|}
\hline & ì & sú & hàabà-báané, & q(à) & $\mathbf{i}$ & ntá & sàaxé & ỳ̀. \\
\hline & $3 P L$ & tous & père-un & mais & $3 P L$ & COPLOC.NEG & mère & POSTP \\
\hline
\end{tabular}

Munaahaxinun lo a do waaxi ke naxan ya, n’i naxan bonondi.

Des hypocrites se sont immiscés entre lui et ce frère et ont gâté leur relation.

\begin{tabular}{|l|l|l|l|l|l|l|l|l|}
\hline & Mùnáahàxí-nù-n & ló & á & dò & wáaxì & ké & nàxá-n & yà, \\
\hline & hypocrite-PL-D & entrer & $3 S G^{\mathrm{H}}$ & avec & frère & $\mathrm{DEM}$ & milieu- $\mathrm{D}^{\mathrm{BH}}$ & $\mathrm{POSTP}$ \\
\hline
\end{tabular}

\begin{tabular}{|l|l|l|l|l|}
\hline & $\mathbf{n}(\grave{a})$ & $\hat{i}$ & nàxá-n & bònò-ndí. \\
\hline & INF & $3 \mathrm{PL}^{\mathrm{H}}$ & milieu-D & \\
\hline
\end{tabular}

I maarentinte yogo d'a qiri koota yi, a ti « An ga d'an yaaxi ke be wara ti munaahaxinun cababun ya, n ya hiibaane konn'an da, an na ken payi, gelli an ga na bonen kita lenki, sere be ga wurunu an ya, ken n'an qananu ku beenu an d'i ga loonanbakken ya, i hana ya hayi wurun'an ya.

Un parent proche à eux l'a appelé un jour en lui disant « Ce frère que tu as abandonné par la faute des hypocrites, je vais te raconter une histoire et tu verras que s'il t'arrive un malheur aujourd'hui, ceux qui te fuiront sont ces amis avec qui tu traînes, ce sont eux qui les premiers te fuiront. 


\begin{tabular}{|l|l|l|l|l|l|l|l|l|}
\hline & I & màarèn-tìnté & yògó & $\mathbf{d}(\mathbf{a})$ & à & qírí & kòotá & yì, \\
\hline & $3 \mathrm{PL}$ & parent-proche & INDEF & $\mathrm{TR}$ & $3 \mathrm{SG}$ & appeler & jour & POSTP \\
\hline
\end{tabular}

\begin{tabular}{|l|l|l|l|l|l|l|l|l|l|l|}
\hline & à & tí & « Án & gà & $\mathbf{d}(\mathbf{a})$ & án & yàaxì & ké & bè & wàrá \\
\hline & $3 S G$ & dire & $2 S G$ & $S B D$ & $T R$ & $2 S G$ & frère & $D^{2} M^{\mathrm{BH}}$ & $\mathrm{REL}$ & laisser \\
\hline
\end{tabular}

\begin{tabular}{|l|l|l|l|l|}
\hline & tî́ & mùnáahàxí-nù-n & càbàbú-n & yà, \\
\hline & avec & hypocrite-PL-D & cause-D & \\
\hline
\end{tabular}

\begin{tabular}{|l|l|l|l|l|l|l|}
\hline & ń & yá & híi-báané & kònn(ó) & án & dà, \\
\hline & 1SG & INACP & affaire-un & dire.GER & 2SG & pour \\
\hline
\end{tabular}

\begin{tabular}{|l|l|l|l|l|l|l|l|l|l|l|}
\hline & án & ná & kén & pàyì, & gèllíi án & gà & ná & bòné-n & kìtà \\
\hline & $2 S G$ & PROJ & DEM & regarder & si & $2 S G$ & SBD & PROJ & malheur-D & obtenir \\
\hline
\end{tabular}

\begin{tabular}{|l|l|l|l|l|l|l|l|}
\hline & lènkí, & sèré & bè & gá & wùrù-nú & án & yà, \\
\hline & aujourd'hui & personne & REL & SBD & courir-GER & 2SG & POSTP \\
\hline
\end{tabular}

\begin{tabular}{|l|l|l|l|l|l|l|}
\hline & kén & $\mathbf{n}(\mathbf{i})$ & án & qànà-nù & kú & bèe-nú \\
\hline & DEM & COPEQ & $2 S G$ & ami-PL & DEM.PL ${ }^{\mathrm{BH}}$ & REL-PL \\
\hline
\end{tabular}

\begin{tabular}{|l|l|l|l|l|l|l|}
\hline & án & $\mathbf{d}(\mathbf{o})$ & ì & gá & lóo-nán-bákkè-n & yá, \\
\hline & $2 S G$ & avec & $3 \mathrm{PL}$ & SBD.COPLOC & entrer-INF-sortir.NMLS-D & POSTP \\
\hline
\end{tabular}

\begin{tabular}{|l|l|l|l|l|l|l|l|}
\hline & í & hàná & yà & háyíi & wùrù-n(ú) & án & yà. \\
\hline & $3 \mathrm{PL}^{\mathrm{H}}$ & premier $^{\mathrm{BH}}$ & $\mathrm{FOC}$ & $\mathrm{OST}$ & courir-GER & $2 \mathrm{SG}$ & POSTP \\
\hline
\end{tabular}

An yaaxibure ke be ga no, har'an ga na bone kita xunbane xunbane halle aken nta wurun'an ya.

Ce mauvais frère que tu as là, même s'il t'arrive un malheur, demain ou plus tard, lui ne te fuira pas.

\begin{tabular}{|l|l|l|l|l|l|l|}
\hline & Án & yàaxì-bùrè & ké & bè & gá & nò, \\
\hline
\end{tabular}




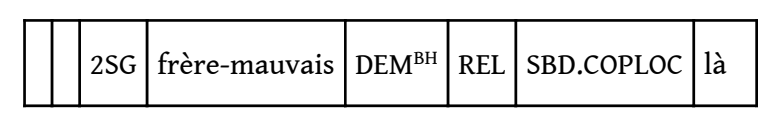

\begin{tabular}{|l|l|l|l|l|l|l|l|l|l|}
\hline & hár(i) & án & gà & ná & bòné & kìtà & xùnbàné, & xùnbàné & hàllé \\
\hline & même & 2SG & SBD & PROJ & malheur & obtenir & demain & demain & après \\
\hline
\end{tabular}

\begin{tabular}{|l|l|l|l|l|l|}
\hline & à-kên & ntá & wùrù-n(ù) & án & yà. \\
\hline & 3SG-EMPH & INACP.NEG & courir-GER & 2SG & POSTP \\
\hline
\end{tabular}

Daga an na selinge kari, an n'a horen yutu an n'a suus'an doroken ya.

Va, tu tueras un poulet, tu prendras son sang et tu le frotteras sur tes habits.

\begin{tabular}{|l|l|l|l|l|l|l|l|l|l|l|}
\hline & Dàgá & án & ná & sélinyé & kàrí, & án & n(á) & á & hòré-n & yùtú \\
\hline & aller & $2 S G$ & PROJ & poulet & tuer & $2 S G$ & $P R O J$ & $3 S^{\mathrm{H}}$ & sang- ${ }^{\mathrm{BH}}$ & prendre \\
\hline
\end{tabular}

\begin{tabular}{|l|l|l|l|l|l|l|l|}
\hline & án & $\mathbf{n}(\mathbf{a})$ & à & sùus(á) & án & dòròkén & yà. \\
\hline & 2SG & PROJ & $3 S G$ & frotter & $2 S G$ & habit-D & POSTP \\
\hline
\end{tabular}

Qananu ku beenu g'an maxa, an na dag'i wulli, an na ti saasa ken : " $N$ da sere kari. "

Ces amis que tu as, tu iras les éveiller, et alors tu leur diras: "J'ai tué quelqu'un.'

\begin{tabular}{|l|l|l|l|l|l|l|}
\hline & Qàná-nú & kú & bèe-nú & g(à) & án & màxá, \\
\hline & ami-PL & DEM.PL & REL-PL & SBD.COPLOC & 2SG & SPHP \\
\hline
\end{tabular}

\begin{tabular}{|c|c|c|c|c|}
\hline án & ná & dàg(á) & ì & wùllí, \\
\hline $2 S G$ & PROJ & aller & $3 P L$ & éveiller \\
\hline
\end{tabular}

\begin{tabular}{|l|l|l|l|l|l|l|l|l|l|}
\hline & án & ná & tì & sáasá & kèn, & « N & dà & sèré & kàrí. » \\
\hline & 2SG & PROJ & dire & maintenant & vraiment & $1 S G$ & TR & personne & tuer \\
\hline
\end{tabular}

I ga na bugu t'an da kille be ya, an na ken yari. ”

Tu verras de quelle façon ils se comportent envers toi. »

\begin{tabular}{|l|l|l|l|l|l|l|l|l|l|l|}
\hline & Ì & gà & ná & bùgù & t(i) & án & dà & killé & bè & yá, \\
\hline
\end{tabular}




\begin{tabular}{|l|l|l|l|l|l|l|l|l|l|l|}
\hline & 3PL & SBD & PROJ & sortir $^{\mathrm{B}}$ & avec & 2SG & pour & chemin & REL & POSTP \\
\hline
\end{tabular}

\begin{tabular}{|c|c|c|c|}
\hline án & ná & kén & yàrí." \\
\hline $2 S G$ & PROJ & DEM & voir \\
\hline
\end{tabular}

A ri, a d'a ña kundun ya, a da selingen kari n'a horen cuusa i doroken ya.

Il est venu et il a fait ainsi, il a tué un poulet et a frotté son sang sur ses habits.

\begin{tabular}{|l|l|l|l|l|l|l|l|l|}
\hline & À & rí, & à & $\mathbf{d}(\mathbf{a})$ & à & ñá & kùndún & yà, \\
\hline & $3 S G$ & venir & $3 S G$ & TR & $3 S G$ & faire & ainsi & POSTP \\
\hline
\end{tabular}

\begin{tabular}{|l|l|l|l|l|}
\hline & à & dà & sélìnjé-n & kàrí \\
\hline & $3 S G$ & TR & poulet-D & tuer \\
\hline
\end{tabular}

\begin{tabular}{|l|l|l|l|l|l|l|l|}
\hline & n(à) & á & hòré-n & cùusá & í & dòròké-n & yà. \\
\hline & INF & $3 S G^{H}$ & sang-D & frotter & REFL & habit-D & POSTP \\
\hline
\end{tabular}

Wuron kaanana, a daga menjanpanan banye, a ti, « Kaatihulaana » a ti, «Giri de, n deema, $n$ da seren ña kari saasa. An m'a yari ? N da labon ña r'a yi, a ma tony'a yi, o na me deema. »

En pleine nuit, il s'est rendu chez un premier ami et lui a dit, « Untel, lève-toi donc, aide-moi, je viens de tuer quelqu'un. Ne vois-tu pas? Je lui ai planté un couteau dans le corps, le coup a été fatal, nous devons nous aider. »

\begin{tabular}{|l|l|l|l|l|l|l|}
\hline & Wùró-n & kàanàanâ, & à & dàgá & mènjàn-pánà-n & bànyé, \\
\hline & nuit-D & milieu_de_nuit. $\mathrm{D}^{\mathrm{BH}}$ & $3 \mathrm{SG}$ & aller & ami-premier-D & côtée $^{\mathrm{BH}}$ \\
\hline
\end{tabular}

\begin{tabular}{|l|l|l|l|l|l|l|l|}
\hline & à & tí, & « Kàatíhùláanà » & à & tí, & « Gìrí & dé, \\
\hline & $3 S G$ & dire & Untel & $3 S G$ & dire & se_lever & EMPH \\
\hline
\end{tabular}

\begin{tabular}{|l|l|l|l|l|l|l|l|l|}
\hline & ń & dèemá, & ń & dà & sèré-n & ñà & kàrì & sáasá. \\
\hline & $1 S G$ & aider & $1 S G$ & TR & personne-D & FOC & tuer $^{\mathrm{B}}$ & maintenant \\
\hline
\end{tabular}

\begin{tabular}{|l|l|l|l|l|l|l|l|l|l|l|l|}
\hline & Án & m(á) & à & yàrì ? & Ń & dà & làbó-n & ñà & r(ò) & à & yí, \\
\hline
\end{tabular}




\begin{tabular}{|l|l|l|l|l|l|l|l|l|l|l|l|}
\hline & $2 S G$ & ACP.NEG & $3 S G$ & voir $^{\mathrm{B}}$ & 1SG & TR & couteau-D & FOC & mettre & 3SG & POSTP \\
\hline
\end{tabular}

\begin{tabular}{|c|c|c|c|c|c|c|c|c|}
\hline à & má & tòny(ò) & à & yí, & ó & nà & mé & dèemá. » \\
\hline $3 S G$ & ACP.NEG & être contenu ${ }^{\mathrm{B}}$ & $3 S G$ & POSTP & 1PL & SUBJ & RECI"P & aider \\
\hline
\end{tabular}

A ti «Ee!» a ti «A ga kiñe serekallen ña yi ken, ike yinmun nta ken yure, an ga heera be dabarini, ken dabari de!

L'ami dit «Ee, vraiment s'il y a eu mort d'homme, je ne me mêle pas de ça, débrouille-toi comme tu peux!

\begin{tabular}{|l|l|l|l|l|l|}
\hline & À & tî̀ & « Ée ! » & à & tí̀ \\
\hline & $3 S G$ & dire & INTERJ & $3 S G$ & dire \\
\hline
\end{tabular}

\begin{tabular}{|l|l|l|l|l|l|l|l|}
\hline & « À & gà & kìné & sèrè-kàllé-n & nà̀ & yí & kèn, \\
\hline & $3 S G$ & SBD & atteindre & personne-mort-D & FOC & POSTP & vraiment \\
\hline
\end{tabular}

\begin{tabular}{|l|l|l|l|l|l|}
\hline & în-ké & yìnmû-n & ntá & kén & yùré, \\
\hline & 1SG-EMPH & tête.PL-D & COPLOC.NEG & DEM & sous \\
\hline
\end{tabular}

\begin{tabular}{|l|l|l|l|l|l|l|l|l|}
\hline & án & gà & héerà & bé & dàbárì-ní, & kén & dàbárì & dé ! \\
\hline & $2 S G$ & SBD & stratagème & REL & faire-GER & DEM & faire & EMPH \\
\hline
\end{tabular}

Ken ga he, i ga n'an kita, i n'an karini ya. "

Sinon, si les gens réussissent à t'attraper, ils te tueront. »

\begin{tabular}{|l|l|l|l|l|l|l|l|l|}
\hline & Kén & gà & hé, & ì & gà & n(á) & án & kìtà, \\
\hline & DEM & SBD & COPEQ.NEG & $3 \mathrm{PL}$ & SBD & PROJ & $2 \mathrm{SG}$ & obtenir \\
\hline
\end{tabular}

\begin{tabular}{|l|l|l|l|l|l|}
\hline & ì & n(à) & án & kàrì-ní & yà. » \\
\hline & $3 P L$ & INACP & $2 S G$ & tuer-GER & FOC \\
\hline
\end{tabular}

A giri nan daga menjanpillandin qa banye, a ti « Hulaana, Hulaana, giri, $n$ da sere kari, an n'in deema. "

Il est parti et s'est rendu encore chez un deuxième ami, et lui a dit « Untel, Untel, lève-toi, j'ai tué quelqu'un, tu dois m'aider. » 


\begin{tabular}{|l|l|l|l|l|l|l|l|}
\hline & À & gìrí & nàn & dàgá & mènjàn-pìllàndí-n & qá & bànyé, \\
\hline & $3 S G$ & se_lever & INF & aller & ami-deuxième-D & aussi & côtéé $^{\text {BH }}$ \\
\hline
\end{tabular}

\begin{tabular}{|c|c|c|c|c|}
\hline à & tí & « Hùláanà, & Hùláanà, & gìrí, \\
\hline $3 S G$ & dire & Untel & Untel & se_lever \\
\hline
\end{tabular}

\begin{tabular}{|l|l|l|l|l|l|l|l|l|}
\hline & ń & dà & sèré & kàrí, & án & n(à) & ín & dèemá. » \\
\hline & $1 S G$ & $T R$ & personne & tuer & $2 S G$ & SUBJ & $1 S G$ & aider \\
\hline
\end{tabular}

"Aa! An t'an da seren kari, ayiwa, ike yinmun nta ken yure, an ga heera be dabarini, ken dabari de!

«Aa, tu dis que tu as tué quelqu'un, eh bien, je ne me mêle pas de ça, débrouille-toi comme tu peux!

\begin{tabular}{|l|l|l|l|l|l|l|l|}
\hline & « Àa ! & Án & $\mathbf{t}(\mathbf{i})$ & án & dà & sèré-n & kàrí, \\
\hline & INTERJ & $2 S G$ & dire & $2 S G$ & TR & personne-D & tuer \\
\hline
\end{tabular}

\begin{tabular}{|l|l|l|l|l|l|l|}
\hline & áyíwà, & í-ké & yìnmû-n & ntá & kén & yùré, \\
\hline & INTERJ & REFL-EMPH & tête.PL-D & COPLOC.NEG & DEM & sous \\
\hline
\end{tabular}

\begin{tabular}{|l|l|l|l|l|l|l|l|l|}
\hline & án & gà & héerà & bé & dàbárìní, & kén & dàbárì & dé ! \\
\hline & $2 S G$ & $S B D$ & stratagème & REL & faire-GER & DEM & faire & EMPH \\
\hline
\end{tabular}

Ken ga he, i ga n'an kita, i n'an karini ya."

Sinon, si les gens réussissent à t'attraper, ils te tueront. »

\begin{tabular}{|l|l|l|l|l|l|l|l|l|}
\hline & Kén & gà & hé, & ì & gà & n(á) & án & kìtà, \\
\hline & DEM & SBD & COPEQ.NEG & $3 \mathrm{PL}$ & $\mathrm{SBD}$ & $\mathrm{PROJ}$ & $2 \mathrm{SG}$ & obtenir $^{\mathrm{B}}$ \\
\hline
\end{tabular}

\begin{tabular}{|c|c|c|c|c|}
\hline ì & n(à) & án & kàrì-ní & yà." \\
\hline 3PL & INACP & $2 S G$ & tuer-GER & FOC \\
\hline
\end{tabular}

A daga katti waaxi ke be a d'a ga haabe, a ti « N gida, n gida, giri!» 
Il est allé vers son frère de même père en lui disant «Grand-frère, grandfrère, lève-toi !»

\begin{tabular}{|l|l|l|l|l|l|l|l|l|l|l|l|}
\hline & À & dàgá & káttì & wáaxì & ké & bè & á & d(ò) & à & gá & hàabé, \\
\hline & $3 S G$ & aller & vers & frère & DEM & REL & $3 S G H$ & avec & $3 S G$ & SBD.COPLOC & père \\
\hline
\end{tabular}

\begin{tabular}{|l|l|l|l|l|l|l|l|}
\hline & à & tí & « $\mathbf{N}$ & gìdá, & ń & gìdá, & gìní ! » \\
\hline & $3 S G$ & dire & $1 S G$ & aîné $^{B H}$ & $1 S G$ & aînéBH & se_lever \\
\hline
\end{tabular}

A ti « Mani ni ? » A ti « N da seren ña kari.»

Le frère a dit « Qu'est-ce que c'est ? » Il a répondu « J'ai tué quelqu'un. »

\begin{tabular}{|l|l|l|l|l|l|l|l|l|l|l|l|}
\hline & À & tî̀ & « Màní & nì ? " & À & tí & « Ń & dà & sèré-n & ñà & kàrì. » \\
\hline & $3 S G$ & dire & quoi ? & COPEQ & $3 S G$ & dire & $1 S G$ & TR & personne-D & FOC & tuer $^{\mathrm{B}}$ \\
\hline
\end{tabular}

A ti «An ga da sere kari? » A ti « Yabo!»

Le frère a demandé « Tu as tué quelqu'un ? » Il a répondu « Oui !»

\begin{tabular}{|l|l|l|l|l|l|l|l|l|l|l|}
\hline & À & tí & « Án & gà & dà & sèré & kàrí ? » & À & tí & « Yàbó ! » \\
\hline & $3 S G$ & dire & $2 S G$ & SBD & TR & personne & tuer & $3 S G$ & dire & oui \\
\hline
\end{tabular}

A ti «A ña kan moxo?» A bugu t’i moxon ya.

Le frère a demandé « Comment ça s'est passé ? » Il a dit ce qui lui était arrivé.

\begin{tabular}{|l|l|l|l|l|l|l|}
\hline & À & tî̀ & « À & ná & kán & mòxó ? " \\
\hline & $3 S G$ & dire & $3 S G$ & se_produire & quel ? & manière \\
\hline
\end{tabular}

\begin{tabular}{|l|l|l|l|l|l|l|}
\hline & À & búgú & $\mathbf{t}(\mathbf{i})$ & $\hat{\mathbf{i}}$ & mòxó-n & yà. \\
\hline & $3 S G$ & sortir & avec & REFL & manière-D & \\
\hline
\end{tabular}

A ti « Saasa ken, ò̀ daga, n qoxone, ò̀ daga ya de!

Le frère a dit «En tout cas maintenant, il faut y aller, petit-frère, il faut vraiment y aller!

\begin{tabular}{|l|l|l|l|l|l|l|l|l|l|}
\hline & À & tî̀ & «Sáasá & kèn, & ó & ǹ & dàgá, & ń & qòxònê, \\
\hline
\end{tabular}




\begin{tabular}{|l|l|l|l|l|l|l|l|l|l|}
\hline & $3 S G$ & dire & maintenant & vraiment & $1 \mathrm{PL}$ & SUBJ & aller & $1 \mathrm{SG}$ & cadet. $\mathrm{D}^{\mathrm{BH}}$ \\
\hline
\end{tabular}

\begin{tabular}{|l|l|l|l|l|l|}
\hline & ó & ǹ & dàgá & yà & dé ! \\
\hline & $1 P L$ & SUBJ & aller & FOC & EMPH \\
\hline
\end{tabular}

I ga na leg'o yi noqqu be yi, i n'o su kari no. "

Si les gens nous rattrapent, ils nous tueront sur-le-champ. »

\begin{tabular}{|l|l|l|l|l|l|l|l|l|l|}
\hline & I & gà & ná & lèg(è) & ó & yì & nòqqú & bè & yí, \\
\hline & $3 \mathrm{PL}$ & $\mathrm{SBD}$ & $\mathrm{PROJ}$ & rattraper $^{\mathrm{B}}$ & $1 \mathrm{PL}$ & $\mathrm{POSTP}$ & endroit & REL & POSTP \\
\hline
\end{tabular}

\begin{tabular}{|l|l|l|l|l|l|l|}
\hline & ì & n(á) & ó & sú & kàrí & nò. " \\
\hline & $3 P L$ & PROJ & 1PL & tous & tuer & endroit \\
\hline
\end{tabular}

Ken noqqu ya ni, i ga kiñe kanpallun ya, a ti « N gida, sigi !» A sigi.

C'est alors que, comme ils arrivaient derrière la maison, il a dit «Grandfrère, arrête-toi ! » Le grand-frère s'est arrêté.

\begin{tabular}{|l|l|l|l|l|l|l|l|l|l|}
\hline & Kén & nòqqú & yà & ní, & ì & gà & kìné & kà-n-pállù-n & yá, \\
\hline & DEM & endroit & FOC & COPEQ & $3 \mathrm{PL}$ & $\mathrm{SBD}$ & atteindre & maison-EP-arrière.PL-D & POSTP \\
\hline
\end{tabular}

\begin{tabular}{|l|l|l|l|l|l|l|l|}
\hline & à & tí & « N & gìdá, & sígí ! » & À & sígí. \\
\hline & $3 S G$ & dire & $1 S G$ & grand-frère $^{\mathrm{BH}}$ & s'arrêter & $3 \mathrm{SG}$ & s'arrêter \\
\hline
\end{tabular}

A ti «N ma hooho ña, an killun taaxundi, $n$ ma hari baane ña.

Il lui a dit « Je n'ai rien fait du tout, rassure-toi, je n'ai rien fait du tout.

\begin{tabular}{|l|l|l|l|l|l|l|}
\hline & À & tí & « Ń & má & hóohò & ñà, \\
\hline & $3 S G$ & dire & $1 S G$ & ACP.NEG & quoi_que_ce_soit & faire $^{\mathrm{B}}$ \\
\hline
\end{tabular}

\begin{tabular}{|c|c|c|c|c|c|c|c|}
\hline án & kìllûu-n & táaxú-ndí, & ń & má & hárì & báané & ñà, \\
\hline $2 S G$ & esprit-D $^{\mathrm{BH}}$ & s'asseoir-CAUS & $1 \mathrm{SG}$ & ACP.NEG & même & un & faire ${ }^{B}$ \\
\hline
\end{tabular}

Yogo yan da ke digaame ko in da nan ti, i ga tini, menjanciren nan pasu waaxiburen ya, i d'a ñi gaaren ña ni ken digaame yi. 
C'est quelqu'un qui m'a dit que quand on dit qu'un bon ami vaut mieux qu'un mauvais frère, c'est faux.

\begin{tabular}{|l|l|l|l|l|l|l|l|l|}
\hline & Yògó & yà-n & dà & ké & dígáamé & kò & în & dà \\
\hline & INDEF & FOC-S & TR & DEM & parole & dire & $1 S G$ & pour \\
\hline
\end{tabular}

\begin{tabular}{|l|l|l|l|l|l|}
\hline & nàn & tí, & $\mathbf{i}$ & gá & tì-ní, \\
\hline & INF & dire & 3PL & SBD.COPLOC & dire-GER \\
\hline
\end{tabular}

\begin{tabular}{|l|l|l|l|l|l|}
\hline & mènjàn-círè-n & nàn & pàsú & wáaxí-búrè-n & yá, \\
\hline & ami-bon-D & ST & valoir_mieux & frère-mauvais-D & POSTP \\
\hline
\end{tabular}

\begin{tabular}{|l|l|l|l|l|l|l|l|l|l|l|}
\hline & ì & $\mathbf{d}(\mathbf{a})$ & à & ñí & gàaré-n & ñà & ní & kén & digàamé & yì. \\
\hline & $3 \mathrm{PL}$ & $\mathrm{TR}$ & $3 \mathrm{SG}$ & trouver & mensonge-D & FOC & COPEQ & DEM & parole & POSTP \\
\hline
\end{tabular}

Ayiwa, $n$ ga dag'in menjanyu ku beenu sagata, baane su t'in danya, $n$ ga ma wuru su, i ga n'in kita su, i n'in karini ya.

Eh bien, quand je suis allé voir mes amis, ils m'ont tous dit que si jamais je ne m'enfuis pas et que les gens m'attrapent, ils me tueront.

\begin{tabular}{|l|l|l|l|l|l|l|l|l|l|}
\hline & Ayíwà, & ń & gà & dàg(á) & în & mènjànyù & kú & bèe-nú & ságátá, \\
\hline & INTERJ & $1 S G$ & SBD & aller & $1 S G$ & ami-PL & DEM.PL ${ }^{\mathrm{BH}}$ & REL-PL & rejoindre \\
\hline
\end{tabular}

\begin{tabular}{|l|l|l|l|l|l|l|l|l|l|l|}
\hline & báané & sú & $\mathbf{t}(\mathbf{i})$ & ín & dànyá, & ń & gà & má & wùrù & sú, \\
\hline & un & tous & dire & $1 S G$ & pour & $1 S G$ & SBD & ACP.NEG & courir $^{B}$ & si_jamais \\
\hline
\end{tabular}

\begin{tabular}{|l|l|l|l|l|l|l|l|l|l|l|l|}
\hline & $\mathbf{i}$ & gà & n(á) & ín & kìtà & sú, & ì & n(à) & ín & kàrì-ní & yà. \\
\hline & $3 \mathrm{PL}$ & $\mathrm{SBD}$ & $\mathrm{PROJ}$ & $1 \mathrm{SG}$ & obtenir $^{\mathrm{B}}$ & si_jamais & $3 \mathrm{PL}$ & $\mathrm{INACP}$ & $1 \mathrm{SG}$ & tuer-GER & FOC \\
\hline
\end{tabular}

Ayiwa, keeta ken, nke d'anke, o ya ni baane ma duna gan giri, munaahaxi su maxa ware o naxa."

Eh bien, vraiment, dorénavant, toi et moi, on reste unis jusqu'à la fin du monde, aucun hypocrite ne doit se glisser entre nous. »

\begin{tabular}{|l|l|l|l|l|l|l|}
\hline & Áyíwà, & kéetá & kèn, & ń-ké & d(ò) & án-kè, \\
\hline
\end{tabular}




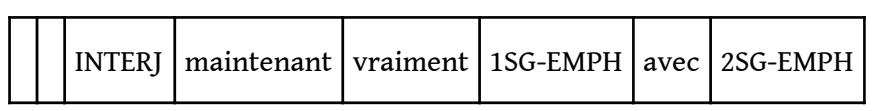

\begin{tabular}{|l|l|l|l|l|l|l|l|l|}
\hline & ó & yà & ní & báané & mà & dúná & gà-n & gìí, \\
\hline & $1 P L$ & FOC & COPEQ & un & jusqu'à & monde & SBD-SUBJ & se_lever \\
\hline
\end{tabular}

\begin{tabular}{|l|l|l|l|l|l|l|}
\hline & mùnáahàxí & sú & máxà & wàré & ó & nàxá. " \\
\hline & hypocrite & tous & PROH & laisser.DETR & $1 \mathrm{PL}$ & $\mathrm{milieu}^{\mathrm{BH}}$ \\
\hline
\end{tabular}

Ken ña ni, sere be d'i waaxin ga ma gemu, munaahaxinun ñan loono qa naxan ya.

En effet, si une personne et son frère ne s'entendent pas, les hypocrites se glisseront entre eux.

\begin{tabular}{|c|c|c|}
\hline Kén & ñà & ní, \\
\hline DEM & FOC & COPEQ \\
\hline
\end{tabular}

\begin{tabular}{|l|l|l|l|l|l|l|l|l|}
\hline & sèré & bè & $\mathbf{d}(\mathbf{o})$ & $\mathbf{i}$ & wàaxí-n & gà & má & gèmù, \\
\hline & personne & REL & avec & REFL & frère-D & SBD & ACP.NEG & s'entendre ${ }^{\mathrm{B}}$ \\
\hline
\end{tabular}

\begin{tabular}{|l|l|l|l|l|l|l|}
\hline & mùnáahàxí-nù-n & ñá-n & lòo-nò & qá & nàxá-n & yà. \\
\hline & hypocrite-PL-D & FOC-S & entrer-GER & aussi & milieu-D ${ }^{\mathrm{BH}}$ & POSTP \\
\hline
\end{tabular}

\subsection{Conte 5 : L'ingratitude}

Ke ña yaxarinqase yogo ya yi.

C'est l'histoire d'une vieille femme.

\begin{tabular}{|l|l|l|l|l|l|l|}
\hline & Ké & ná & yàxàrì-n-qásé & yògó & yà & yí. \\
\hline & DEM & être & femme-EP-vieux & INDEF & FOC & POSTP \\
\hline
\end{tabular}

Tunkayugobaane wa deben kaaran ya.

Il y a un roi aux environs du village.

\begin{tabular}{|l|l|l|l|l|l|}
\hline & Tùnkà-yùgò-báané & wá & dèbé-n & kàará-n & yà. \\
\hline & roi-homme-un & COPLOC & village-D & côté-D ${ }^{\mathrm{BH}}$ & POSTP \\
\hline
\end{tabular}


Ken tunkayugo, yillen n'a baane ya maxa, a g'a gaagana.

Ce roi, c'est lui seul qui a du mil à vendre.

\begin{tabular}{|l|l|l|l|l|l|l|l|l|}
\hline & Kén & tùnkà-yúgó, & yìllé-n & n(à) & á & bàané & yà & màxá, \\
\hline & DEM & roi-homme & mil-D & COPLOC & $3 S^{\mathrm{H}}$ & $\mathrm{un}^{\mathrm{BH}}$ & FOC & $\mathrm{SPHP}$ \\
\hline
\end{tabular}

\begin{tabular}{|l|l|l|l|l|}
\hline & à & g(á) & à & gáagà-ná. \\
\hline & $3 S G$ & SBD.INACP & $3 S G$ & vendre-GER \\
\hline
\end{tabular}

Yille su nta jamaane ke yi, qa i be ga na daga katti ken tunkayugo yi, t'an ya yillen qobono, a n'an tirindini ya, duna ga lagarini moxo be yi.

Il n'y avait pas du tout de mil dans le pays, mais si quelqu'un allait trouver ce roi en lui disant qu'on voulait acheter du mil, il lui demandait comment le monde finirait.

\begin{tabular}{|l|l|l|l|l|l|l|}
\hline & Yìllé & sú & ntá & jàmáanè & ké & yì, \\
\hline & mil & aucun & COPLOC.NEG & pays & DEM & POSTP \\
\hline
\end{tabular}

\begin{tabular}{|l|l|l|l|l|l|l|l|l|l|l|}
\hline & qà & $\hat{\mathbf{i}}$ & bè & gà & ná & dàgà & káttì & kén & tùnkà-yúgó & yì, \\
\hline & mais & REFL & REL & SBD & PROJ & aller & vers & DEM & roi-homme & POSTP \\
\hline
\end{tabular}

\begin{tabular}{|l|l|l|l|l|l|}
\hline & $\mathbf{t}(\mathbf{i})$ & án & yá & yillê-n & qóbó-nó, \\
\hline & dire & $2 S G$ & COPLOC & mil-D & acheter-GER \\
\hline
\end{tabular}

\begin{tabular}{|l|l|l|l|l|l|}
\hline & à & n(à) & án & tírìndì-ní & yà, \\
\hline & $3 S G$ & INACP & $2 S G$ & interroger-GER & FOC \\
\hline
\end{tabular}

\begin{tabular}{|l|l|l|l|l|l|l|}
\hline & dúná & gà & làgàrì-ní & mòxó & bè & yí. \\
\hline & monde & SBD & finir-GER & manière & REL & POSTP \\
\hline
\end{tabular}

I be ga n'a tu, a na yillen gaag'an ya, an ga m'a tu, a n'an karini ya.

Si la personne en question savait, il lui vendait du mil, si elle ne savait pas, il la tuait.

\begin{tabular}{|l|l|l|l|l|l|l|}
\hline II & bè & gà & n(á) & à & tù, \\
\hline
\end{tabular}




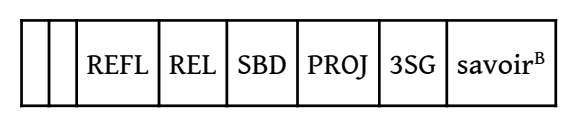

\begin{tabular}{|c|c|c|c|c|c|}
\hline à & ná & yìllêe-n & gáag(à) & án & yà, \\
\hline $3 S G$ & PROJ & mil-D & vendre & $2 S G$ & POSTP \\
\hline
\end{tabular}

\begin{tabular}{|l|l|l|l|l|l|l|l|l|l|l|}
\hline & án & gà & $\mathbf{m}(\mathbf{a})$ & à & tù, & à & n(à) & án & kàrì-ní & yà. \\
\hline & 2SG & SBD & ACP.NEG & $3 S G$ & savoir $^{B}$ & $3 S G$ & INACP & 2SG & tuer-GER & FOC \\
\hline
\end{tabular}

Yaxarinqase yogo giri, t'ike wa dagana.

Une vieille femme a décidé d'y aller.

\begin{tabular}{|l|l|l|l|l|l|l|l|}
\hline & Yàxàrì-n-qásé & yògó & gìní, & t(ì) & î-ké & wá & dàgà-ná. \\
\hline & femme-EP-vieux & INDEF & se_lever & dire & REFL-EMPH & INACP & aller-GER \\
\hline
\end{tabular}

I ti « An ga na daga, an ga na kori digaame ke jaabin konno, a n'an karini ya. »

Les gens lui ont dit «Si tu y vas et que tu échoues à dire la réponse à cette parole, il te tuera.»

\begin{tabular}{|c|c|c|c|c|c|}
\hline I & t(i) & « Án & gà & ná & dàgà, \\
\hline $3 \mathrm{PL}$ & dire & $2 S G$ & SBD & PROJ & aller $^{\mathrm{B}}$ \\
\hline
\end{tabular}

\begin{tabular}{|l|l|l|l|l|l|l|l|l|}
\hline & án & gà & ná & kòrì & digàamé & ké & jàabín & kònnó, \\
\hline & $2 S G$ & $S B D$ & $P R O J$ & échouer $^{\mathrm{B}}$ & parole & $\mathrm{DEM}$ & ${\text { réponse- } \mathrm{D}^{\mathrm{BH}}}^{\mathrm{N}}$ & dire.GER \\
\hline
\end{tabular}

\begin{tabular}{|c|c|c|c|c|}
\hline à & n(à) & án & kàrì-ní & yà. " \\
\hline $3 S G$ & INACP & $2 S G$ & tuer-GER & FOC \\
\hline
\end{tabular}

A ti «Yabo, a ga n'in kari, a w'a ñiin'in caaten ña ni. »

Elle a dit « Oui, s'il me tue, ce sera parce que c'était ma destinée. »

\begin{tabular}{|l|l|l|l|l|l|l|l|l|}
\hline & À & tí & « Yàbó, & à & gà & n(á) & ín & kàrì, \\
\hline & $3 S G$ & dire & oui & $3 S G$ & SBD & PROJ & 1SG & tuer $^{B}$ \\
\hline
\end{tabular}

\begin{tabular}{|l|l|l|l|l|l|l|l|l|}
\hline & à & w(á) & à & ñìi-n(í) & în & càatén & ñà & ní. " \\
\hline
\end{tabular}




\begin{tabular}{|l|l|l|l|l|l|l|l|l|}
\hline & & $3 S G$ & COPLOC & $3 S G$ & trouver-GER & 1SG & destinée-D & \\
\hline
\end{tabular}

A do kisimaren daga, i ga dangini baane tunguron ya, kuuto yogo wa saqqa tungurunkunmen ya.

Elle est partie avec son petit-fils, et comme ils passaient seulement devant une termitière, il y avait un varan allongé à l'entrée de la termitière.

\begin{tabular}{|l|l|l|l|l|}
\hline & Á & dò & kìsìmàré-n & dàgá, \\
\hline & $3 S G^{\mathrm{H}}$ & avec & petit_fils-D & aller \\
\hline
\end{tabular}

\begin{tabular}{|l|l|l|l|l|l|l|}
\hline & ì & gá & dángì-ní & bàané & túngúrò-n & yá, \\
\hline & $3 \mathrm{PL}$ & SBD.INACP & passer-GER & seulement & termitière-D & POSTP \\
\hline
\end{tabular}

\begin{tabular}{|l|l|l|l|l|l|l|}
\hline & kúutó & yògó & wá & sáqqá & túngúrú-n-kúnmè-n & yá. \\
\hline & varan & INDEF & INACP & s'allonger.GER & termitière-EP-trou-D & POSTP \\
\hline
\end{tabular}

A da yaxarinqase ke qiri, $t$ ' "An dagana minna?»

Il a appelé la vieille et lui a demandé « Où vas-tu? »

\begin{tabular}{|l|l|l|l|l|l|l|l|l|l|}
\hline & À & dà & yàxàrì-n-qásé & ké & qírí, & t(î) & « Án & dàgàná & mínnà ? » \\
\hline & $3 S G$ & TR & femme-EP-vieux & DEM & appeler & dire & $2 S G$ & aller-GER & où? \\
\hline
\end{tabular}

A ti i dagana ke tunkayugo ya banye i qa t'a na seren tirindini hon ñogo ya yi, an ga n'a ko, a na yillen gaag'an ya, an ga m'a ko, a n'an kari.

Elle a dit qu'elle allait chez le roi dont on raconte qu'il pose une question aux gens, et que si ils trouvent la réponse, il leur vend du mil, mais si ils ne la trouvent pas, il les tue.

\begin{tabular}{|l|l|l|l|l|l|l|l|l|}
\hline & À & tí & í & dàgà-ná & ké & túnká-yúgó & yà & bànyé \\
\hline & $3 S G$ & dire & REFL & aller-GER & DEM & roi-homme & FOC & côtébH $^{B H}$ \\
\hline
\end{tabular}

\begin{tabular}{|l|l|l|l|l|l|l|l|}
\hline & ì & qá & $\mathbf{t}(\mathbf{i})$ & à & nà & sèrê-n & tírìndì-ní \\
\hline & $3 P L$ & aussi & dire & $3 S G$ & INACP & personne-D & interroger-GER \\
\hline
\end{tabular}

\begin{tabular}{|l|l|l|l|l|l|l|l|l|l|}
\hline & hó-n & ǹogó & yà & yí, & án & gà & n(á) & à & kò, \\
\hline
\end{tabular}




\begin{tabular}{|l|l|l|l|l|l|l|l|l|l|}
\hline & chose-D & INDEF & FOC & POSTP & 2SG & SBD & PROJ & 3 SS & dire $^{\mathrm{B}}$ \\
\hline
\end{tabular}

\begin{tabular}{|l|l|l|l|l|l|l|}
\hline & à & ná & yìllê-n & gáag(à) & án & yà, \\
\hline & $3 S G$ & PROJ & mil-D & vendre & 2SG & POSTP \\
\hline
\end{tabular}

\begin{tabular}{|l|l|l|l|l|l|l|l|l|l|}
\hline & án & gà & $\mathbf{m}(\mathbf{a})$ & $\mathbf{a}$ & kò, & à & n(á) & án & kàrí. \\
\hline & $2 S G$ & $S B D$ & $A C P . N E G$ & $3 S G$ & $\operatorname{dire}^{B}$ & $3 S G$ & PROJ & $2 S G$ & tuer \\
\hline
\end{tabular}

A ti «An ga na daga, a w'an tirindini, ti duna lagarini kan moxo, an na ti, duna lagarini hiisiringallaaxun ña yi. »

Le varan a dit « Si tu y vas, il te demandera comment le monde finira, alors tu lui diras que c'est par l'ingratitude que le monde finira. »

\begin{tabular}{|l|l|l|l|l|l|l|l|l|l|l|}
\hline & À & tí & « Án & gà & ná & dàgà, & à & w(á) & án & tírìndi-ní, \\
\hline & $3 S G$ & dire & $2 S G$ & SBD & PROJ & aller & $3 S G$ & INACP & $2 S G$ & interroger-GER \\
\hline
\end{tabular}

\begin{tabular}{|l|l|l|l|l|l|l|l|l|}
\hline & tí & dúná & làgàrì-ní & kán & mòxó, & án & ná & tì, \\
\hline & dire & monde & finir-GER & quel ? & manière & 2SG & PROJ & dire \\
\hline
\end{tabular}

\begin{tabular}{|l|l|l|l|l|l|}
\hline & dúná & làgàrì-ní & híisírínyálláaxù-n & ñá & yì. » \\
\hline & monde & finir-GER & ingratitude-D & FOC & POSTP \\
\hline
\end{tabular}

A ga daga katti tunkayugo ke yi, a t'i ri yillixobe ya.

Elle est allée vers le roi et lui a dit qu'elle venait pour acheter du mil.

\begin{tabular}{|l|l|l|l|l|l|l|l|}
\hline & À & gà & dàgá & káttì & tùnkà-yúgó & ké & yì, \\
\hline & $3 S G$ & SBD & aller & vers & roi-homme & DEM & POSTP \\
\hline
\end{tabular}

\begin{tabular}{|c|c|c|c|c|c|}
\hline à & $t(\mathbf{i})$ & í & rí & yillli-xóbé & yà. \\
\hline $3 S G$ & dire & REFL & venir & mil-acheter.DETR & FOC \\
\hline
\end{tabular}

A ti « Diganbaane ya na yere de.

Le roi a dit « Il y a là une parole. 


\begin{tabular}{|l|l|l|l|l|l|l|l|}
\hline & À & tí & « Dìgàn-báané & yà & nà & yèré & dé. \\
\hline & $3 S G$ & dire & parole-un & FOC & COPLOC & ici & EMPH \\
\hline
\end{tabular}

An ga d'a tu, yillen payi, n n'a gaag'an ya.

Si tu la reconnais, voici le mil, je te le vendrai.

\begin{tabular}{|l|l|l|l|l|l|l|l|}
\hline & Án & gà & $\mathbf{d}(\mathbf{a})$ & $\mathbf{a}$ & tú, & yillê-n & páyí, \\
\hline & $2 S G$ & SBD & TR & $3 S G$ & connaître & mil-D & OST \\
\hline
\end{tabular}

\begin{tabular}{|l|l|l|l|l|l|l|}
\hline & ń & n(á) & à & gáag(à) & án & yà. \\
\hline & $1 S G$ & PROJ & $3 S G$ & vendre & $2 S G$ & POSTP \\
\hline
\end{tabular}

An ga m'a tu, kaahan payi, n n'an qurusini ya. » A ti « Yabo. »

Si tu ne la reconnais pas, voici le sabre, je t'égorgerai. » Elle a dit « Oui. »

\begin{tabular}{|l|l|l|l|l|l|l|l|}
\hline & Án & gà & m(á) & à & tù, & káahàn & páyí, \\
\hline & $2 S G$ & SBD & ACP.NEG & $3 S G$ & connaître $^{B}$ & sabre-D & OST \\
\hline
\end{tabular}

\begin{tabular}{|l|l|l|l|l|l|l|l|l|}
\hline & ń & $\mathbf{n}(\mathbf{a})$ & án & qùrúsì-ní & yà. » & À & tí & « Yàbó. " \\
\hline & $1 S G$ & INACP & $2 S G$ & égorger-GER & FOC & $3 S G$ & dire & oui \\
\hline
\end{tabular}

A ti « Ayiwa, duna lagarini kan moxo?

Il dit « Eh bien, comment est-ce que le monde finira?

\begin{tabular}{|l|l|l|l|l|l|l|l|}
\hline & À & tî́ & « Áyíwà, & dúná & làgàrìní & kán & mòxó ? \\
\hline & $3 S G$ & dire & INTERJ & monde & finir-GER & quel ? & manière \\
\hline
\end{tabular}

An na ke baane jaab'in ya. "

C'est seulement à cela que tu dois répondre. »

\begin{tabular}{|l|l|l|l|l|l|l|l|}
\hline & Án & ná & ké & bàané & jáab(i) & în & yà. » \\
\hline & $2 S G$ & PROJ & DEM & un $^{\mathrm{BH}}$ & répondre & $1 \mathrm{SG}$ & POSTP \\
\hline
\end{tabular}

Yaxarinqasen ti, «Duna lagarini hiisiringallaaxun ña yi. » 
La vieille femme a dit « C'est par l'ingratitude que le monde finira. »

\begin{tabular}{|l|l|l|}
\hline & Yàxàrì-n-qásè-n & tí, \\
\hline & femme-EP-vieux-D & dire \\
\hline
\end{tabular}

\begin{tabular}{|l|l|l|l|l|l|}
\hline & « Dúná & làgàrì-ní & híisírínyálláaxù-n & ñá & yì. » \\
\hline & monde & finir-GER & ingratitude-D & FOC & POSTP \\
\hline
\end{tabular}

A ti « Wallaahi, » a ti « Anken d'a kita. »

Le roi a dit «Wallaahi, tu es tombée juste.»

\begin{tabular}{|l|l|l|l|l|l|l|l|l|l|}
\hline & À & tí & « Wàlláahì, » & à & tí & « án-kèn & $\mathbf{d}(\mathbf{a})$ & à & kìtá. » \\
\hline & $3 S G$ & dire & INTERJ & $3 S G$ & dire & 2SG-EMPH & TR & $3 S G$ & obtenir \\
\hline
\end{tabular}

A da yillen gaag'a yi.

Il lui a vendu le mil.

\begin{tabular}{|l|l|l|l|l|l|l|}
\hline & À & dà & yìllê-n & gáag(à) & à & yí. \\
\hline & $3 S G$ & TR & mil-D & vendre & $3 S G$ & POSTP \\
\hline
\end{tabular}

I ga yillene, i ga ri tunguro ke noqqun ya, "A t'i kisimaren danya, a ti, n kisimare, ayiwa, o da nahaxan kita, a toxo mani ya? A toxo tiyen ña.

Sur le chemin du retour, alors qu'ils arrivaient à l'endroit de la termitière, la vieille femme a dit à son petit-fils «Mon petit-fils, d'accord, nous avons trouvé de quoi manger, mais qu'est-ce qui manque? Il manque la viande.

\begin{tabular}{|l|l|l|l|}
\hline & İ & gá & yíllè-né, \\
\hline & 3PL & SBD.COPLOC & retourner-GER \\
\hline
\end{tabular}

\begin{tabular}{|l|l|l|l|l|l|l|l|}
\hline & ì & gà & rí & túngúró & ké & nòqqún & yà, \\
\hline & $3 \mathrm{PL}$ & $\mathrm{SBD}$ & venir & termitière & $\mathrm{DEM}$ & endroit- $\mathrm{D}^{\mathrm{BH}}$ & $\mathrm{POSTP}$ \\
\hline
\end{tabular}

\begin{tabular}{|l|l|l|l|l|l|l|l|}
\hline & $\mathbf{a}$ & $\mathbf{t}(\mathbf{i})$ & $\mathbf{i}$ & kìsìmàrén & dànyá, & $\mathbf{a ̀}$ & tí, \\
\hline & $3 S G$ & dire & REFL & petit-fils- $\mathrm{D}^{\mathrm{BH}}$ & pour & $3 \mathrm{SG}$ & dire \\
\hline
\end{tabular}




\begin{tabular}{|l|l|l|l|l|l|l|l|}
\hline & « N & kìsìmàrê, & áyíwà, & ó & dà & náháxà-n & kìtá, \\
\hline & $1 S G$ & petit-fils- $\mathrm{D}^{\mathrm{BH}}$ & INTERJ & $1 \mathrm{PL}$ & $\mathrm{TR}$ & nourriture & obtenir \\
\hline
\end{tabular}

\begin{tabular}{|l|l|l|l|l|l|l|l|l|}
\hline & à & tóxó & màní & yà ? & À & tóxó & tíyè-n & ñá. \\
\hline & $3 S G$ & rester & quoi ? & FOC & $3 S G$ & rester & viande-D & FOC \\
\hline
\end{tabular}

Tiye wa dangini kuuton ya ba?

Y a-t-il meilleure viande que le varan?

\begin{tabular}{|l|l|l|l|l|l|l|}
\hline & Tíyé & wá & dángì-ní & kúutò-n & yá & bà ? \\
\hline & viande & INACP & passer-GER & varan-D & POSTP & Q \\
\hline
\end{tabular}

O na kuuton laga, o d'a na daga, o n'a kari n'a ña yiraxaten ya. »

Nous allons attraper le varan, l'emporter, le tuer et en faire notre repas. »

\begin{tabular}{|l|l|l|l|l|l|l|l|l|l|}
\hline & o & ná & kúutò-n & lágà, & ó & $\mathbf{d}(\mathbf{o})$ & à & ná & dàgá, \\
\hline & $1 \mathrm{PL}$ & $\mathrm{PROJ}$ & varan-D & attraper & $1 \mathrm{PL}$ & avec & $3 \mathrm{SG}$ & $\mathrm{PROJ}$ & aller \\
\hline
\end{tabular}

\begin{tabular}{|l|l|l|l|l|l|l|l|l|l|}
\hline & ó & $\mathbf{n}(\mathbf{a})$ & $\mathbf{a}$ & kàríi & $\mathbf{n}(\mathbf{a})$ & à & ñá & yìràxátè-n & yá. " \\
\hline & $1 P L$ & PROJ & $3 S G$ & tuer & INF & $3 S G$ & faire & repas-D & POSTP \\
\hline
\end{tabular}

Ken noqqu ya ni, kuuton t'a danya, "Ayiwa, n ga da digaame be ko an danya, ken digaame ya hayi ke kundu, inke yan ña sababu yi, an ga wase kalla, ken ga he, a ñ'an karini ya, qa yelli an d'inke toxono ti ken ya ba.»

C'est là que le varan lui a dit, « Eh bien, ce que je t'avais dit, c'est ce qui s'est réalisé, c'est grâce à moi que tu as échappé à la mort, car autrement il t'aurait tuée, et avec tout ça, est-ce que tu m'as laissé en vie?

\begin{tabular}{|l|l|l|l|l|l|l|l|l|}
\hline & Kén & nòqqú & yà & ní, & kúutò-n & $\mathbf{t}(\mathbf{i})$ & á & dànyá, \\
\hline & DEM & endroit & FOC & COPEQ & varan-D & dire & $3 S G^{\mathrm{H}}$ & pour \\
\hline
\end{tabular}

\begin{tabular}{|l|l|l|l|l|l|l|l|l|l|}
\hline & « Áyíwà, & ń & gà & dà & digàamé & bè & kó & án & dànyá, \\
\hline & INTERJ & $1 S G$ & $\mathrm{SBD}$ & $\mathrm{TR}$ & parole & $\mathrm{REL}$ & dire & $2 \mathrm{SG}$ & pour \\
\hline
\end{tabular}

\begin{tabular}{|l|l|l|l|l|l|l|}
\hline & kén & digàamé & yà & háyí & ké & kúndù, \\
\hline
\end{tabular}




\begin{tabular}{|l|l|l|l|l|l|l|}
\hline & DEM & parole & FOC & OST & DEM & ainsi \\
\hline
\end{tabular}

\begin{tabular}{|l|l|l|l|l|l|l|l|l|l|}
\hline & în-ké & yà-n & nà̀ & sábábù & yí, & án & gà & wàsé & kàllá, \\
\hline & 1SG-EMPH & FOC-S & être $^{\mathrm{B}}$ & cause & POSTP & 2SG & SBD & échapper & mourir.GER \\
\hline
\end{tabular}

\begin{tabular}{|l|l|l|l|l|l|l|l|l|}
\hline & kén & gà & hé, & à & ñ(i) & án & kàrì-ní & yà, \\
\hline & DEM & SBD & COPEQ.NEG & $3 S G$ & PAS & $2 S G$ & tuer-GER & FOC \\
\hline
\end{tabular}

\begin{tabular}{|l|l|l|l|l|l|l|l|l|l|l|}
\hline & qà & yélli & án & $\mathrm{d}(\mathbf{a})$ & ín-ké & tóxó-nó & tì & kén & yà & bá. \\
\hline & mais & pourvu_que & $2 \mathrm{SG}$ & $\mathrm{TR}$ & $1 \mathrm{SG}-\mathrm{EMPH}$ & laisser-GER & avec & DEM & POSTP & $\mathrm{Q}$ \\
\hline
\end{tabular}

A heti an ga da inke hiisiringalla ba?»

N'est-ce pas que tu m'as traité avec ingratitude?»

\begin{tabular}{|l|l|l|l|l|l|l|l|l|}
\hline & À & hètí & án & gà & dà & ín-ké & híisírínyállá & bà ? " \\
\hline & $3 S G$ & COPEQ.NEG & $2 S G$ & SBD & TR & 1SG-EMPH & être_ingrat_envers & Q \\
\hline
\end{tabular}

\section{Proverbes}

An ga da nuxudunyurugumen yari a ga to, a da arahatun ña kita.

Si tu vois que celui qui avait la diarrhée se met à péter, c'est que ça va mieux.

\begin{tabular}{|l|l|l|l|l|l|l|l|l|}
\hline & Án & gà & dà & nùxù-dù-n-yùrù-gùmé-n & yàrí & à & gà & tó, \\
\hline & $2 S G$ & $S B D$ & $T R$ & ventre-LOC-EP-Courir-PROPR-D & voir & $3 S G$ & SBD & péter \\
\hline
\end{tabular}

\begin{tabular}{|l|l|l|l|l|l|}
\hline & à & dà & àràhátù-n & ñá & kìtà. \\
\hline & $3 S G$ & TR & soulagement-D & FOC & obtenir $^{B}$ \\
\hline
\end{tabular}

An ga m'an ma digaamen mugu, an n'an ma tayinnen digaamen mugu.

Si tu n'écoutes pas ce que te dit ta mère, tu écouteras ce que te dit sa coépouse.

\begin{tabular}{|l|l|l|l|l|l|l|l|}
\hline & Án & gà & m(á) & án & mà & digàamé-n & mùgù, \\
\hline
\end{tabular}




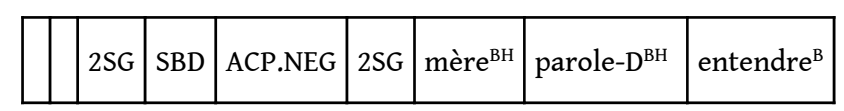

\begin{tabular}{|l|l|l|l|l|l|l|l|}
\hline & án & n(á) & án & mà & tàyìnné-n & digàamé-n & mùgú. \\
\hline & $2 S G$ & PROJ & $2 S G$ & mère ${ }^{\mathrm{BH}}$ & co_épouse- $\mathrm{D}^{\mathrm{BH}}$ & parole- $\mathrm{D}^{\mathrm{BH}}$ & entendre $^{\mathrm{B}}$ \\
\hline
\end{tabular}

An ga n'a mugu ti baaxan lo tagayen naxan ya, a kare ya.

Si tu entends dire qu'un margouillat s'est glissé dans le mur, c'est qu'il était cassé.

\begin{tabular}{|l|l|l|l|l|l|l|l|l|}
\hline & An & gà & n(á) & $\grave{a}$ & mùgù & tí & báaxà-n & ló \\
\hline & $2 S G$ & SBD & PROJ & $3 S G$ & entendre & que & margouillat & entrer \\
\hline
\end{tabular}

\begin{tabular}{|c|c|c|c|c|c|}
\hline tàgáyè-n & nàxá-n & yà, & à & káré & yà. \\
\hline mur-D & milieu-D若 & POSTP & $3 S G$ & casser.DETR & FOC \\
\hline
\end{tabular}

An ga n'an ñaaxun lox'an kallu ya, an na tere biten di.

Si tu prêtes tes yeux à ton beau-père, tu marcheras dans le noir.

\begin{tabular}{|l|l|l|l|l|l|l|l|l|l|}
\hline & Án & gà & n(á) & án & ǹàaû-n & lòx(ò) & án & kàllú & yà, \\
\hline & $2 S G$ & $S B D$ & PROJ & $2 S G$ & œil.PL- $D^{\mathrm{BH}}$ & prêter $^{\mathrm{B}}$ & $2 \mathrm{SG}$ & beau-parent $^{\mathrm{B}}$ & FOC \\
\hline
\end{tabular}

\begin{tabular}{|l|l|l|l|l|l|}
\hline & án & ná & tèré & bìté-n & di. \\
\hline & $2 S G$ & PROJ & marcher & obscurité-D & dans \\
\hline
\end{tabular}

An ga n'an qannen yilla moxo wo moxo do halle yi, an nt'an kohen yalli.

$\mathrm{Tu}$ auras beau tourner ton cou vers l'arrière, tu ne verras pas ta nuque.

\begin{tabular}{|l|l|l|l|l|l|l|l|l|l|}
\hline & Án & gà & $\mathbf{n}(\mathbf{a})$ & án & qànnê-n & yillà & mòxó & wó & mòxò \\
\hline & $2 S G$ & $S B D$ & $P R O J$ & $2 S G$ & Cou- $\mathrm{D}^{\mathrm{BH}}$ & tourner & manière & DISTR & manière \\
\hline
\end{tabular}

\begin{tabular}{|c|c|c|c|c|c|c|c|}
\hline dó & hállè & yí, & án & nt(á) & án & kòhé-n & yàlli. \\
\hline avec & arrière & POSTP & $2 S G$ & INACP.NEG & $2 S G$ & nuque- $\mathrm{D}^{\mathrm{BH}}$ & voir.GER ${ }^{\mathrm{B}}$ \\
\hline
\end{tabular}

Án ga na dalla jarinten moosono ya ni an ga katta a qanma. 
C'est en caressant longuement la lionne qu'on arrive à la traire.

\begin{tabular}{|l|l|l|l|l|l|l|l|l|l|}
\hline & Án & gà & ná & dàllà & jàrìnté-n & mòosò-nó & yà & ní & \\
\hline & $2 S G$ & $S B D$ & PROJ & durer & lion-D & caresser-GER & FOC & COPEQ & \\
\hline
\end{tabular}

\begin{tabular}{|l|l|l|l|l|l|}
\hline & án & gà & káttà & à & qánmá. \\
\hline & $2 S G$ & SBD.INACP & pouvoir.GER & $3 S G$ & traire.GER \\
\hline
\end{tabular}

An ga na dooren qayen morogo moxon be, a kaawana kundun ña yi.

Le banco sèche avec la forme qu'on lui a donnée en le moulant quand il était frais.

\begin{tabular}{|c|c|c|c|c|c|c|c|}
\hline Án & gà & ná & dòoré-n & qàyé-n & mòrògò & mòxó-n & bè, \\
\hline $2 S G$ & SBD & PROJ & banco-D & frais- $\mathrm{D}^{\mathrm{BH}}$ & mouler $^{B}$ & manière-D & REL \\
\hline
\end{tabular}

\begin{tabular}{|l|l|l|l|l|l|}
\hline & à & káawá-ná & kùndún & ñà & yí. \\
\hline & $3 S G$ & sécher-GER & ainsi & FOC & POSTP \\
\hline
\end{tabular}

An ga na gaja be kanbon jaara, a ya n'an qinñi.

Si tu soignes les dents d'une souris, c'est elle qui te mordra.

\begin{tabular}{|l|l|l|l|l|l|l|l|}
\hline & Án & gà & ná & gàjá & bè & kànbó-n & jàarà, \\
\hline & $2 S G$ & $S B D$ & $P R O J$ & souris & $R E L$ & dent.PL- $D^{B H}$ & soigner \\
\hline
\end{tabular}

\begin{tabular}{|l|l|l|l|l|l|}
\hline & á & yà & n(à) & án & qìnñì. \\
\hline & $3 S G^{\mathrm{H}}$ & FOC & INACP & $2 S G$ & mordre.GER \\
\hline
\end{tabular}

An ga na kanu moxo wo moxo, an la nta wurunu n'an tinkanbun toxo.

Même si on a très peur, on ne peut pas s'enfuir en abandonnant ses fesses.

\begin{tabular}{|l|l|l|l|l|l|l|l|}
\hline & Án & gà & ná & kànù & mòxó & wó & mòxò, \\
\hline & $2 S G$ & SBD & PROJ & avoir_peur & manière & DISTR & manière \\
\hline
\end{tabular}

\begin{tabular}{|l|l|l|l|l|l|l|l|l|}
\hline & án & là & ntá & wùrù-nù & n(à) & án & tìnkànbû-n & tóxó. \\
\hline
\end{tabular}




\begin{tabular}{|l|l|l|l|l|l|l|l|l|}
\hline & 2SG & POT & INACP.NEG & courir-GER $^{B}$ & INF & 2SG & fesse.PL-D & laisser \\
\hline
\end{tabular}

An ga na kor'an ma yi, an n'an maama sugu.

Si tu ne peux pas avoir ta mère, tu tèteras ta grand-mère.

\begin{tabular}{|l|l|l|l|l|l|l|l|}
\hline & Án & gà & ná & kòr(i) & án & mà & yí, \\
\hline & $2 S G$ & SBD & PROJ & échouer & $2 S G$ & mère $^{B H}$ & POSTP \\
\hline
\end{tabular}

\begin{tabular}{|l|l|l|l|l|l|}
\hline & án & $\mathbf{n}(a ́)$ & án & màamá & sùgú. \\
\hline & $2 S G$ & PROJ & $2 S G$ & grand-mère ${ }^{\mathrm{BH}}$ & téter \\
\hline
\end{tabular}

An ga na ñi tini, n ñinmen ciyinten ni, a ma kiñe kunande yi, kuhunen bug'a yi!

Si tu dis que tu as la tête rasée, ce n'est pas la peine de jurer, enlève seulement ton chapeau!

\begin{tabular}{|l|l|l|l|l|l|l|l|l|l|}
\hline & Án & gà & ná & ñì & tì-ní, & ń & ñìnmé-n & cì̀̀-nté-n & nì, \\
\hline & $2 S G$ & SBD & PROJ & se_trouver & dire-GER & $1 S G$ & tête-D ${ }^{\mathrm{BH}}$ & raser-RES-D & COPEQ \\
\hline
\end{tabular}

\begin{tabular}{|l|l|l|l|l|l|}
\hline & à & má & kìnè & kùnà-ndé & ỳ̀, \\
\hline & $3 S G$ & ACP.NEG & atteindre $^{B}$ & jurer-NMLS & POSTP \\
\hline
\end{tabular}

\begin{tabular}{|l|l|l|l|l|}
\hline & kúhúnèn & búg(ú) & à & yí ! \\
\hline & chapeau-D & enlever & 3SG & POSTP \\
\hline
\end{tabular}

An ga na sugon liixi, a n'an qiñi.

Si tu pousses une chèvre à bout, elle te mordra.

\begin{tabular}{|l|l|l|l|l|l|l|l|l|l|}
\hline & Án & gà & ná & súgò-n & lìixì, & à & n(á) & án & qíníí. \\
\hline & $2 S G$ & SBD & PROJ & chèvre-D & coincer $^{B}$ & 3SG & PROJ & 2SG & mordre \\
\hline
\end{tabular}

An ga na tayinnen caaten kin'a metayinnen ya, a w'a karini.

Si tu donnes à une épouse d'un mari polygame le moyen de décider de la vie de sa co-épouse, elle la tuera.

\begin{tabular}{|l|l|l|l|l|l|l|}
\hline & Án & gà & ná & tàyìnnê-n & càaté-n & kìn(i) \\
\hline
\end{tabular}


\begin{tabular}{|l|l|l|l|l|l|l|}
\hline & 2SG & SBD & PROJ & co_épouse-D & destinée & donner \\
\hline
\end{tabular}

\begin{tabular}{|l|l|l|l|l|l|l|l|}
\hline & á & mè-tàyìnné-n & yà, & à & w(á) & à & kàrì-ní. \\
\hline & $3 S G^{\mathrm{H}}$ & RECIP-co-épouse- $\mathrm{D}^{\mathrm{BH}}$ & POSTP & $3 \mathrm{SG}$ & INACP & $3 \mathrm{SG}$ & tuer-GER \\
\hline
\end{tabular}

An ga na ti, an nta dagana kaanen an nta toqqo halle, axa soro sikki ya ni.

Si tu dis que tu ne passeras pas devant et que tu ne resteras pas derrière, c'est que vous êtes trois.

\begin{tabular}{|l|l|l|l|l|l|l|l|l|}
\hline & Án & gà & ná & tì, & án & ntá & dàgànà & káanè, \\
\hline & $2 S G$ & SBD & PROJ & dire $^{\mathrm{B}}$ & $2 \mathrm{SG}$ & INACP.NEG & aller-GER $^{\mathrm{B}}$ & devant \\
\hline
\end{tabular}

\begin{tabular}{|l|l|l|l|l|l|l|l|l|l|}
\hline & án & ntá & tòqqò & hállè, & áxá & sòró & sìkkì & yá & nì. \\
\hline & $2 S G$ & INACP.NEG & rester-GER & derrière & $2 \mathrm{PL}$ & personne.PL & trois $^{\mathrm{B}}$ & FOC & COPEQ \\
\hline
\end{tabular}

An ga na ti, an nta saqqa ma naxaane, axa soro sikki ya ni.

Si tu dis que tu ne te coucheras qu'au milieu, c'est que vous êtes trois.

\begin{tabular}{|l|l|l|l|l|l|l|l|l|l|}
\hline & Án & gà & ná & tì, & án & ntá & sàqqà & mà & nàxáanè, \\
\hline & 2SG & SBD & PROJ & dire & 2SG & INACP.NEG & se_coucher.GER & sauf & milieu \\
\hline
\end{tabular}

\begin{tabular}{|l|l|l|l|l|l|l|l|}
\hline & áxá & sòró & sìkkì & yá & nì. & & \\
\hline & $2 \mathrm{PL}$ & personne.PL & trois $^{\mathrm{B}}$ & FOC & COPEQ & & \\
\hline
\end{tabular}

An ga nexu janban ya, a siiti an jon ña di.

Si tu sous-estimes un complot, c'est qu'il a été tramé en ta présence.

\begin{tabular}{|l|l|l|l|l|l|}
\hline & Án & gà & nèxú & jànbá-n & yà, \\
\hline & 2SG & SBD & sous_estimer & comploit-D & POSTP \\
\hline
\end{tabular}

\begin{tabular}{|l|l|l|l|l|l|l|}
\hline & à & s̀ìíi & án & jòn & ñá & dì \\
\hline & 3SG & être_attaché & $2 S G$ & devant-D & FOC & dans \\
\hline
\end{tabular}

An la wa tini « Kaati n'in qanaxasen ya, » qa « Kaati n'in yaaxixasen ya », ken ni garen ña yi. 
$\mathrm{Tu}$ peux dire «Untel est mon ancien ami », mais «Untel est mon ancien frère ", c'est un mensonge.

\begin{tabular}{|l|l|l|l|l|l|l|l|l|l|}
\hline & Án & là & wá & tì-ní & « Kàatí & n(i) & în & qànà-xàsé-n & yà, " \\
\hline & $2 S G$ & POT & INACP & dire-GER & Untel & COPEQ & $1 S G$ & ami-ancien-D ${ }^{\mathrm{BH}}$ & POSTP \\
\hline
\end{tabular}

\begin{tabular}{|l|l|l|l|l|l|l|}
\hline & qà & « Kàatíi & $\mathbf{n}(\mathbf{i})$ & ín & yàaxì-xàsé-n & yà, " \\
\hline & mais & Untel & COPEQ & 1 SG & frère-ancien-D & POSTP \\
\hline
\end{tabular}

\begin{tabular}{|l|l|l|l|l|l|}
\hline & kén & nì & gàaré-n & ñà & yí. \\
\hline & DEM & COPEQ & mensonge-D & FOC & POSTP \\
\hline
\end{tabular}

An paaba ga da doroke qob'an da, duruxoto na jiiba mund'a ya!

Si ton père t'achète un boubou, efforce-toi de lui trouver une poche!

\begin{tabular}{|l|l|l|l|l|l|l|l|l|}
\hline & Án & pàabá & gà & dà & dòròké & qób(ó) & án & dà, \\
\hline & $2 S G$ & père $^{\mathrm{BH}}$ & $\mathrm{SBD}$ & $\mathrm{TR}$ & boubou & acheter & $2 \mathrm{SG}$ & pour \\
\hline
\end{tabular}

\begin{tabular}{|l|l|l|l|l|l|l|l|}
\hline & dù & dòxótò & nà & jíibà & mùnd(ú) & à & yí ! \\
\hline & REFL & s'efforcer & INF & poche & trouver & $3 S G$ & POSTP \\
\hline
\end{tabular}

Baanaaxun nan ciri Alla baane ya yi.

La solitude n'est bonne que pour Dieu.

\begin{tabular}{|l|l|l|l|l|l|l|l|}
\hline & Báanáaxù-n & nàn & cír(i) & Állà & bàané & yà & yí. \\
\hline & solitude-D & ST & être_bon & Dieu & un $^{\mathrm{BH}}$ & FOC & POSTP \\
\hline
\end{tabular}

Baanaaxun da Alla baane ya naha.

La solitude n'est utile qu'à Dieu.

\begin{tabular}{|l|l|l|l|l|l|l|l|}
\hline & Báanáaxùn & dà & Állà & bàané & yà & nàhà. & \\
\hline & solitude-D & TR & Dieu & un $^{\mathrm{BH}}$ & FOC & servir & \\
\hline
\end{tabular}

Baananteraanan ga na t'ike da Ala yari, ko qa n'a kutana? 
Si un voyageur solitaire dit qu'il a rencontré Dieu, qui va le contredire?

\begin{tabular}{|l|l|l|l|l|l|l|l|l|l|}
\hline & Báaná-n-téráanà-n & gà & ná & $\mathbf{t}(\mathbf{i})$ & î-ké & dà & Alà & yàrí, \\
\hline & un-EP-voyageur-D & SBD & PROJ & dire & REFL-EMPH & TR & Dieu & voir \\
\hline
\end{tabular}

\begin{tabular}{|l|l|l|l|l|l|}
\hline & kó & qá & n(à) & à & kùtà-nà ? \\
\hline & qui ? & aussi & INACP & $3 S G$ & contredire-GER \\
\hline
\end{tabular}

Benne rege taro yi, a t'i jootan ña ni.

La corne a atteint la taille de l'oreille, elle dit qu'elle a le même âge.

\begin{tabular}{|l|l|l|l|l|}
\hline & Bénné & régè & táró & ỳ̀, \\
\hline & corne & rattraper & oreille & POSTP \\
\hline
\end{tabular}

\begin{tabular}{|l|l|l|l|l|l|l|}
\hline & à & $\mathbf{t}(\mathbf{i})$ & í & jòotá-n & ñà & ní. \\
\hline & $3 S G$ & dire & REFL & personne_de_même_âge-D & FOC & COPEQ \\
\hline
\end{tabular}

Beranqullen cu heti qurura yi.

Tous les endroits bien propres ne sont pas des endroits pour faire ses besoins.

\begin{tabular}{|l|l|l|l|l|l|}
\hline & Bérá-n-qúllè-n & cú & hètí & qùrùrá & yì. \\
\hline & place-EP-blanc-D & tous & COPEQ.NEG & endroit_pour_déféquer & POSTP \\
\hline
\end{tabular}

Berejun ñan kappa me yi n'a ña dugaanen ya.

Ce sont les petits tas de gerbes qui forment la meule.

\begin{tabular}{|l|l|l|l|l|l|}
\hline & Béréjù-n & ñá-n & kàppà & mé & ỳ̀ \\
\hline & petit_tas_de_gerbes.PL-D & FOC-D & s'unir.GER & RECIP & POSTP \\
\hline
\end{tabular}

\begin{tabular}{|l|l|l|l|l|l|}
\hline & n(à) & à & ñá & dùgàané-n & yà. \\
\hline & INF & $3 S G$ & devenir & meule-D & POSTP \\
\hline
\end{tabular}

Booton duuron la nta sikki. 
Un sac ne tient pas vide en position verticale.

\begin{tabular}{|l|l|l|l|l|l|}
\hline & Bòotó-n & dùuró-n & là & ntá & sìkkì. \\
\hline & sac-D & vide- $\mathrm{D}^{\mathrm{BH}}$ & POT & INACP.NEG & se_dresser.GER \\
\hline
\end{tabular}

Dagaanan nta qurusire qurunu.

Celui qui part ne fait pas un bon excrément.

\begin{tabular}{|l|l|l|l|l|}
\hline & Dàg-áanà-n & ntá & qùrù-síré & qùrù-nù \\
\hline & partir-NMAG-D & INACP & excrément-bon & déféquer-GER \\
\hline
\end{tabular}

Daganancaagayen na hunen ña da, ken palle giden nta bakka $i$ batten di.

Le va-et-vient n'intéresse que le singe, sinon la colline ne quitte pas sa place.

\begin{tabular}{|l|l|l|l|l|l|}
\hline & Dàgà-nàn-càagá-yè-n & nà & hùné-n & naà & dá, \\
\hline & aller-INF-repartir-NMLS-D & COPLOC & singe-D & FOC & pour \\
\hline
\end{tabular}

\begin{tabular}{|c|c|c|c|c|c|c|c|}
\hline kén & pàllé & gídè-n & ntá & bàkkà & î́ & bàtté-n & di \\
\hline DEM & derrière & colline-D & INACP.NEG & sortir.GER ${ }^{\mathrm{B}}$ & REFL & place- $\mathrm{D}^{\mathrm{BH}}$ & dans \\
\hline
\end{tabular}

Diganparanparen do tonyun peti baane yi.

Une belle parole et la vérité, ce n'est pas la même chose.

\begin{tabular}{|l|l|l|l|l|l|l|}
\hline & Dìgàn-páránpárè-n & dó & tònyú-n & pètí & báané & yì. \\
\hline & parole-beau-D & avec & vérité-D & COPEQ.NEG & un & POSTP \\
\hline
\end{tabular}

Diminnan ya hunen ya tiganten noxon ya bawo jiiba nt'a yi.

Le singe a de l'amertume dans le champ d'arachide car il n'a pas de poche.

\begin{tabular}{|l|l|l|l|l|l|l|l|}
\hline & Dímínnà-n & yá & hùné-n & yà & tìgà-n-té-n & nòxó-n & dì \\
\hline & amertume-D & COPLOC & singe- D & POSTP & arachide-EP-champ-D & intérieur-D & dans \\
\hline
\end{tabular}

\begin{tabular}{|l|l|l|l|l|l|}
\hline & báwò & jíibá & nt(á) & à & yí. \\
\hline
\end{tabular}




\begin{tabular}{|l|l|l|l|l|l|}
\hline & car & poche & COPLOC.NEG & 3SG & POSTP \\
\hline
\end{tabular}

Dooren qayen ñan tagene.

C'est quand il est frais qu'on moule le banco.

\begin{tabular}{|c|c|c|c|}
\hline Dòoré-n & qàyé-n & ñà-n & tàgè-nè. \\
\hline banco-D & frais- $\mathrm{D}^{\mathrm{BH}}$ & FOC-S & façonner.DETR-GER \\
\hline
\end{tabular}

Dudoxotoyen la wa jarintenqatin tagana seren danya.

La persévérance peut procurer du lait de lionne.

\begin{tabular}{|l|l|l|l|l|l|}
\hline & Dù-dòxótò-yé-n & là & wá & jàrìntè-n-qátì-n & tàgà-ná \\
\hline & REFL-s'efforcer.NMLS-D & POT & INACP & lion-EP-lait-D & créer-GER \\
\hline
\end{tabular}

\begin{tabular}{|l|l|l|}
\hline & sèré-n & dànyá. \\
\hline & personne-D & pour \\
\hline
\end{tabular}

Dukoyintaaxun ñan da sugon qoqqen toxo halle.

C'est seulement pour se faire voir que la chèvre a sa queue au derrière.

\begin{tabular}{|l|l|l|l|l|l|l|l|}
\hline & Dù-kòyì-nt-àaxú-n & ñà-n & dà & súgò-n & qòqqé-n & tòxò & hállè. \\
\hline & REFL-montrer-RES-ABSTR-D & FOC-S & TR & chèvre-D & queue-D $\mathrm{D}^{\mathrm{BH}}$ & laisser & derrière \\
\hline
\end{tabular}

Dullinten nta sange tu.

Un affamé ne connaît pas la plaisanterie.

\begin{tabular}{|l|l|l|l|l|}
\hline & Dúllí-ntè-n & ntá & sángé & tù. \\
\hline & avoir_faim-RES-D & INACP.NEG & jeu & connaître.GER \\
\hline
\end{tabular}

Duna ga na bono moxo wo moxo, muusinen nta saqqa yelle kanma.

Le monde a beau être gâté, le chat ne couve pas des œufs.

\begin{tabular}{|l|l|l|l|l|l|l|l|}
\hline & Dúná & gà & ná & bònò & mòxó & wó & mòxò, \\
\hline & monde & SBD & PROJ & s'abîmer & manière & DISTR & manière $^{\mathrm{B}}$ \\
\hline
\end{tabular}




\begin{tabular}{|c|c|c|c|c|}
\hline múusìnê-n & ntá & sàqqà & yèllé & kànmá. \\
\hline chat-D & INACP.NEG & se_coucher.GER ${ }^{B}$ & œuf & sur \\
\hline
\end{tabular}

Gaaren ga na wuru moxo wo moxo, tonyun y'a ragana.

Le mensonge a beau courir, la vérité le rattrape.

\begin{tabular}{|l|l|l|l|l|l|l|l|}
\hline & Gàaré-n & gà & ná & wùrù & mòxó & wó & mòxò, \\
\hline & mensonge-D & SBD & PROJ & courir $^{B}$ & manière & DISTR & manière $^{\mathrm{B}}$ \\
\hline
\end{tabular}

\begin{tabular}{|l|l|l|l|l|}
\hline & tònyû-n & $\mathbf{y}(\mathbf{a})$ & $\mathbf{a}$ & rágà-ná. \\
\hline & vérité-D & $\mathrm{m}$ & $3 \mathrm{SG}$ & attraper-GER \\
\hline
\end{tabular}

Gannin jin ntaxa geden di.

L'eau d'autrefois n'est plus dans le puits.

\begin{tabular}{|l|l|l|l|l|l|}
\hline & Gánnín & jì-n & ntáxà & gèdé-n & dì. \\
\hline & autrefois & eau-D & COPLOC.CESS & puits-D & dans \\
\hline
\end{tabular}

Guruda ga na dalla moxo wo moxo jin di, a nta ñaana ñexe yi.

Aussi longtemps qu'une souche reste dans l'eau, elle ne se transforme pas en poisson.

\begin{tabular}{|l|l|l|l|l|l|l|l|l|l|}
\hline & Gùrùdá & gà & ná & dàllà & mòxó & wó & mòxò & jín & di, \\
\hline & souche & SBD & PROJ & durer $^{\mathrm{B}}$ & manière & DISTR & manière & eau-D & dans \\
\hline
\end{tabular}

\begin{tabular}{|l|l|l|l|l|l|}
\hline & à & ntá & ñàa-nà & ñéxé & yì. \\
\hline & 3SG & INACP.NEG & devenir $^{B}$ & poisson & POSTP \\
\hline
\end{tabular}

Hankaman paaba kayin'a halle ya.

C'est en l'absence du souverain qu'on insulte son père.

\begin{tabular}{|c|c|c|c|c|c|}
\hline Hànkàmá-n & pàabá & kà-yì-n(i) & á & hàllé & yà. \\
\hline souverain-D & père ${ }^{\mathrm{BH}}$ & insulter-DETR-GER & $3 \mathrm{SG}^{\mathrm{H}}$ & derrière & FOC \\
\hline
\end{tabular}

Hantanyen $t i$ «ji, » q'a ma ti « jintaye. » 
Le crapaud a parlé d'eau, pas d'eau chaude.

\begin{tabular}{|l|l|l|l|l|l|l|l|l|}
\hline & Hàntányè-n & $\mathbf{t i ́}$ & «jî, » & $\mathbf{q}(\mathbf{a})$ & à & má & tì & «jí-n-tàyê. » \\
\hline & crapaud-D & dire & eau & mais & $3 S G$ & ACP.NEG & dire ${ }^{\mathrm{B}}$ & eau-EP-chaud.D \\
\hline
\end{tabular}

Haren ga n'an danbu, an qa ga n'a danbu, axa su n'baana.

Si un âne te donne un coup de patte et que tu lui donnes aussi un coup de pied, vous êtes pareils.

\begin{tabular}{|l|l|l|l|l|l|}
\hline & Hàré-n & gà & n(á) & án & dànbù, \\
\hline & âne-D & SBD & PROJ & $2 S G$ & donner_un_coup_de_pied \\
\hline
\end{tabular}

\begin{tabular}{|l|l|l|l|l|l|l|}
\hline & án & qá & gà & n(á) & à & dànbù, \\
\hline & $2 S G$ & aussi & SBD & PROJ & $3 S G$ & donner_un_coup_de_pied \\
\hline
\end{tabular}

\begin{tabular}{|c|c|c|c|}
\hline áxá & sú & n(àn) & báanà. \\
\hline $2 \mathrm{PL}$ & tous & ST & être identique \\
\hline
\end{tabular}

Hari huren ga laaxara, a nda dalla no, a w'i saxumoxon tuunu.

Même le défunt dans l'au-delà, au bout d'un certain temps il sait comment s'allonger.

\begin{tabular}{|l|l|l|l|l|l|l|l|l|}
\hline & Hárì & hùré-n & gà & láaxàrá, & à & ndá & dàllà & nó, \\
\hline & même & défunt-D & SBD.COPLOC & l'au-delà & $3 S G$ & SBD.PROJ & durer $^{B}$ & là \\
\hline
\end{tabular}

\begin{tabular}{|l|l|l|l|l|l|}
\hline & à & w(á) & $\hat{\mathbf{i}}$ & sàxù-mòxó-n & tùu-nú. \\
\hline & $3 S G$ & INACP & REFL & s'allonger-manière-D & connaître-GER \\
\hline
\end{tabular}

Hiisiringallen kuyen ni yiraame ya yi.

Le meilleur cadeau qu'on puisse offrir à un ingrat est un pagne.

\begin{tabular}{|c|c|c|c|c|c|}
\hline Híisírínyállè-n & kùyé-n & nì & yìràamé & yà & yí. \\
\hline ingrat-D & cadeau- $\mathrm{D}^{\mathrm{BH}}$ & COPEQ & pagne & FOC & POSTP \\
\hline
\end{tabular}

Hinkinten y'a mund'i yaqqen na maxaholinge soro diina tooreharanpare yi. 
L'aveugle est plus intéressé par le fait que sa femme cuisine bien que par le fait qu'elle ait de belles tresses.

\begin{tabular}{|l|l|l|l|l|l|l|l|}
\hline & Hìnkìntê-n & $\mathbf{y}(\mathbf{a})$ & à & mùnd(á) & $\hat{\mathbf{i}}$ & yàqqé-n & nà \\
\hline & aveugle-D & INACP & $3 S \mathrm{SG}$ & vouloir.GER & REFL & épouse-D & \\
\hline $\mathrm{BH}$ & SUBJ \\
\hline
\end{tabular}

\begin{tabular}{|l|l|l|l|l|l|}
\hline & màxàhò-línyé & sòró & díinà & tóoré-háránpáré & yì. \\
\hline & sauce-bon & cuisiner & plutôt_que & tresse-beau & POSTP \\
\hline
\end{tabular}

Hunen ga na suuñi moxo wo moxo, a ra nt'a ñaana qoyi hadamarenme.

Le singe a beau essayer de se moucher, il ne peut pas le faire comme un être humain.

\begin{tabular}{|l|l|l|l|l|l|l|l|}
\hline & Hùné-n & gà & ná & sùuñì & mòxó & wó & mòxò, \\
\hline & singe-D & SBD & PROJ & se_moucher & manière & DISTR & manière $^{B}$ \\
\hline
\end{tabular}

\begin{tabular}{|l|l|l|l|l|l|l|l|}
\hline & à & rà & nt(á) & à & ñàa-nà & qóyì & hádámárénmè. \\
\hline & $3 S G$ & POT & INACP.NEG & $3 S G$ & faire-GER & Comme & être_humain.D \\
\hline
\end{tabular}

Hure ga na muxu i yanqindaanon ya, a do mexen ñan dagana laaxara.

Si un cadavre se cache de ceux qui font sa toilette, il partira sale dans l'autre monde.

\begin{tabular}{|l|l|l|l|l|l|l|l|}
\hline & Hùré-n & gà & ná & mùxù & í & yànqìndàanó-n & yà, \\
\hline & cadavre-D & SBD & PROJ & se_cacher & REFL & laveur.PL-D ${ }^{\mathrm{BH}}$ & POSTP \\
\hline
\end{tabular}

\begin{tabular}{|l|l|l|l|l|l|l|}
\hline & á & dò & méxè-n & ñá-n & dàgà-nà & láaxàrá \\
\hline & $3 S G^{\mathrm{H}}$ & avec & saleté-D & FOC-S & partir-GER & l'au_delà \\
\hline
\end{tabular}

I be nda tiiden muñi, an na qoren kita.

Qui supporte la fumée aura les braises.

\begin{tabular}{|l|l|l|l|l|l|l|l|l|l|}
\hline & I & bè & ndá & tìidé-n & mùñì, & án & ná & qórè-n & kìtá. \\
\hline & REFL & REL & SBD.PROJ & fumée-D & supporter $^{\mathrm{B}}$ & $2 \mathrm{SG}$ & PROJ & braise-D & obtenir \\
\hline
\end{tabular}


I ga ti "qanaaxu», turunye ti "kootabaane qanaaxu, ken ma qoto, har'inke ra wa ken danya.»

Quand on a parlé d'amitié l'hyène a dit, «L'amitié d'un jour, ce n'est pas difficile, même moi je peux faire ça ».

\begin{tabular}{|l|l|l|l|l|l|l|}
\hline & I & gà & tì & « qànàaxû, » & túrúnyé & tì \\
\hline & $3 \mathrm{PL}$ & $\mathrm{SBD}$ & dire & amitié & hyène & dire \\
\hline
\end{tabular}

\begin{tabular}{|l|l|l|l|l|l|}
\hline & « kòotà-báané & qànàaxû, & kên & má & qòtò, \\
\hline jour-un & amitié $^{\mathrm{BH}}$ & DEM & ACP.NEG & être_dur \\
\hline
\end{tabular}

\begin{tabular}{|l|l|l|l|l|l|l|}
\hline & hár(i) & în-ké & rà & wá & kén & dànyá » \\
\hline & même & 1SG-EMPH & POT & COPLOC & DEM & pour \\
\hline
\end{tabular}

I ga ti « yaaxen ya yigene, » $i$ ma ti « a qaye.»

Si on dit qu'on mange les yeux, ça ne veut pas dire qu'on les mange crus.

\begin{tabular}{|l|l|l|l|l|l|l|}
\hline & I & gà & tí & « yáaxè-n & yá & yígé-né, " \\
\hline & $3 \mathrm{PL}$ & $\mathrm{SBD}$ & dire & œil-D & INACP & manger.DETR-GER \\
\hline
\end{tabular}

\begin{tabular}{|l|l|l|l|l|l|l|}
\hline & ì & má & tì & « á & qàyê. » & \\
\hline & $3 \mathrm{PL}$ & ACP.NEG & $\operatorname{dire}^{\mathrm{B}}$ & $3 \mathrm{SG}^{\mathrm{H}}$ & $\mathrm{cru}^{\mathrm{BH}}$ & \\
\hline
\end{tabular}

I ti « digaame, manin d'an bonondi ? » a ti « $i$ konimoxo », i ti « manin d'an cirondi ? » a ti « $i$ konimoxo.»

« Parole, qu'est-ce qui t'a gâtée ? » «La façon de me dire. » «Qu'est-ce qui t'a améliorée? » « La façon de me dire. »

\begin{tabular}{|l|l|l|l|l|l|l|l|}
\hline & I & tí & « digàamê, & màní-n & $\mathbf{d}(\mathbf{a})$ & án & bònò-ndi ? » \\
\hline & $3 \mathrm{PL}$ & dire & parole-D & quoi ?-S & TR & $2 \mathrm{SG}$ & s'abîmer-CAUS \\
\hline
\end{tabular}

\begin{tabular}{|l|l|l|l|l|}
\hline & à & tí & «î́ & kònì-mòxô. " \\
\hline & $3 S G$ & dire & REFL & dire-manière- $\mathrm{D}^{\mathrm{BH}}$ \\
\hline
\end{tabular}

\begin{tabular}{|l|l|l|l|l|l|l|}
\hline & İ & tí & « màní-n & $d(a ̀)$ & án & cìrò-ndì ? " \\
\hline
\end{tabular}




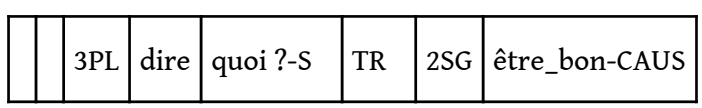

\begin{tabular}{|l|l|l|l|l|}
\hline & à & tí & «í & kònì-mòxô. " \\
\hline & $3 S G$ & dire & REFL & dire-manière- $\mathrm{D}^{\mathrm{BH}}$ \\
\hline
\end{tabular}

I ti « dorokun payi juman ya, » samaqqe ti « $i$ wa kunkigumun da. »

On dit qu'il y a des boubous à la mosquée, le serpent dit qu'ils sont pour ceux qui ont des épaules.

\begin{tabular}{|l|l|l|l|l|l|l|}
\hline & I & tí & « dòròkûn & páyí júmà-n & yá, " \\
\hline & 3PL & dire & boubou.PL & OST & mosquée-D & POSTP \\
\hline
\end{tabular}

\begin{tabular}{|l|l|l|l|l|l|l|}
\hline & sámáqqé & tì & «ì & wá & kúnkí-gúmù-n & dá. » \\
\hline & serpent & dire & 3PL & COPLOC & épaule-PROPR.PL-D & pour \\
\hline
\end{tabular}

Jamankahoncugo, abada a wuyini gunnen ña yi.

La chèvre qui a plusieurs maîtres passe toujours la nuit en brousse.

\begin{tabular}{|l|l|}
\hline & Jàmà-n-kàhò-n-cúgò, \\
\hline & groupe_de_gens-EP-association-EP-chèvre.D \\
\hline
\end{tabular}

\begin{tabular}{|l|l|l|l|l|l|l|l|}
\hline & ábádá & à & wùyì-ní & gùnné-n & ñà & yí. & \\
\hline & toujours & $3 S G$ & passer_la_nuit-GER & brousse-D & FOC & POSTP & \\
\hline
\end{tabular}

Ji be ga joxi ra ntaxa qoorene. = Jiijoxinten la nta qoorene.

L'eau versée ne se ramasse plus / ne peut pas se ramasser.

\begin{tabular}{|l|l|l|l|l|l|l|l|}
\hline & Jí & bè & gà & jòxí & rà & ntáxà & qóoré-né. \\
\hline & eau & REL & SBD & verser.DETR & POT & CESS & ramasser.DETR-GER \\
\hline
\end{tabular}

\begin{tabular}{|l|l|l|l|l|}
\hline & Jiii-jóxí-ntè-n & là & ntá & qòorè-nè. \\
\hline & eau-verser.DETR-RES-D & POT & INACP.NEG & ramasser.DETR-GER \\
\hline
\end{tabular}

Jin ga tawo moxo wo moxo, a murono ya. 
Si chaude que soit l'eau, elle se refroidira.

\begin{tabular}{|l|l|l|l|l|l|l|}
\hline & Jí-n & gà & táwó & mòxó & wó & mòxò, \\
\hline & eau-D & SBD & se_réchauffer & manière & DISTR & manière $^{\mathrm{B}}$ \\
\hline
\end{tabular}

\begin{tabular}{|l|l|l|l|}
\hline & à & mùrò-nó & yà. \\
\hline & $3 S G$ & se_refroidir- GER & FOC \\
\hline
\end{tabular}

Jinmullen la wa jintayen murondini.

L'eau froide peut refroidir l'eau chaude.

\begin{tabular}{|l|l|l|l|l|l|}
\hline & Jí-n-mùllé-n & là & wá & jí-n-tàyé-n & mùrò-ndi-ní. \\
\hline & eau-EP-froid-D & POT & INACP & eau-EP-chaud-D & se_refroidir-CAUS-GER \\
\hline
\end{tabular}

Jintawu hilli nta me murondini.

Deux eaux chaudes ne se refroidissent pas l'une l'autre.

\begin{tabular}{|c|c|c|c|c|}
\hline Jí-n-tàwú & hilli & ntá & mé & mùrò-ndì-nì. \\
\hline eau-EP-chaud.PL & $\operatorname{deu} x^{B}$ & INACP.NEG & RECIP & se_refroidir-CAUS-GER ${ }^{B}$ \\
\hline
\end{tabular}

Kanmen yure jabaren nta tuyini.

Sous la pluie on ne remarque pas la transpiration.

\begin{tabular}{|l|l|l|l|l|l|}
\hline & Kànmé-n & yùré & jàbàrê-n & ntá & tù-yì-nì. \\
\hline & pluie-D & sous & transpiration-D & INACP.NEG & connaître-DETR-GER \\
\hline
\end{tabular}

Kanmen yure yaaxanjin nta tuyini.

Sous la pluie on ne remarque pas les larmes.

\begin{tabular}{|l|l|l|l|l|l|}
\hline & Kànmé-n & yùré & yáaxá-n-jìin & ntá & tù-yì-nì. \\
\hline & pluie-D & sous & œil-EP-eau-D & INACP.NEG & connaître-DETR-GER ${ }^{\mathrm{B}}$ \\
\hline
\end{tabular}

Katt'an ya jigin'an qenuran ya, jig'an taloran ya!

Au lieu d'accuser l'endroit où tu es tombé, accuse l'endroit où tu as trébuché. 


\begin{tabular}{|l|l|l|l|l|l|l|l|}
\hline & Kátt(i) & án & yá & jììi-n(i) & án & qènù-rá-n & yà, \\
\hline & avant_que & $2 S G$ & INACP & tenir_pour_responsable-GER & 2SG & tomber-lieu-D ${ }^{\mathrm{BH}}$ & POSTP \\
\hline
\end{tabular}

\begin{tabular}{|l|l|l|l|l|}
\hline & jìg(i) & án & tàlò-rá-n & yà ! \\
\hline & tenir_pour_responsable & $2 S G$ & trébucher-lieu-D & \\
\hline
\end{tabular}

Kennen ga kalla walle be, a nt'a danqannen mukku.

La pintade n'entend pas le tambour qui sonne la battue où elle mourra.

\begin{tabular}{|l|l|l|l|l|l|}
\hline & Kènné-n & gà & kàllá & wàllé & bè, \\
\hline & pintade-D & SBD.COPLOC & mourir.GER & battue & REL \\
\hline
\end{tabular}

\begin{tabular}{|l|l|l|l|l|l|}
\hline & à & nt(á) & á & dàn-qànné-n & mùkkù. \\
\hline & $3 S G$ & INACP.NEG & $3 S G^{\mathrm{H}}$ & tambour-son- $\mathrm{D}^{\mathrm{BH}}$ & entendre.GER \\
\hline
\end{tabular}

Kiiden dinkoyen do i moxo wo moxo, a ma kati hari labunqare bagandini.

La grosseur du baobab ne lui a même pas permis de fournir un manche de couteau.

\begin{tabular}{|l|l|l|l|l|l|l|l|}
\hline & Kìidé-n & dinkòyé-n & dò & í & mòxó & wó & mòxò, \\
\hline & baobab-D & grosseur-D $\mathrm{D}^{\mathrm{BH}}$ & avec & REFL & manière $^{\mathrm{BH}}$ & DISTR & manière $^{\mathrm{B}}$ \\
\hline
\end{tabular}

\begin{tabular}{|l|l|l|l|l|l|l|}
\hline & à & má & kàtì & hárì & làbù-n-qáré & búgá-ndí-ní. \\
\hline & 3SG & ACP.NEG & pouvoir $^{B}$ & même & couteau-EP-manche & sortir-CAUS-GER \\
\hline
\end{tabular}

Kiiden nan dara, qa a gingen nta ñaana labunqare yi.

Le baobab est gros, mais sa branche ne fait pas un manche de couteau.

\begin{tabular}{|l|l|l|l|}
\hline & Kìidé-n & nàn & dàrá, \\
\hline & baobab-D & ST & être_gros \\
\hline
\end{tabular}

\begin{tabular}{|l|l|l|l|l|l|l|l|}
\hline & qà & á & gìngé-n & ntá & ñàa-nà & làbù-n-qáré & yì. \\
\hline & mais & $3 S G^{\mathrm{H}}$ & branche- $\mathrm{D}^{\mathrm{BH}}$ & INACP & devenir-GER & couteau-EP-manche & POSTP \\
\hline
\end{tabular}


Killen ga laato wo laato, a warene debe ya kanma.

Si longue que soit la route, elle aboutit à un village.

\begin{tabular}{|l|l|l|l|l|l|}
\hline & Killé-n & gà & láató & wó & làatò, \\
\hline & route-D & SBD & s'éloigner & CONC & s'éloigner \\
\hline
\end{tabular}

\begin{tabular}{|l|l|l|l|l|l|}
\hline & à & wàrè-né & dèbé & yà & kànmá. \\
\hline & $3 S G$ & laisser.DETR-GER & village & FOC & sur \\
\hline
\end{tabular}

Lemine nta marene mukkun liikoota.

On n'attend pas l'arrivée des étrangers pour éduquer un enfant.

\begin{tabular}{|l|l|l|l|l|l|}
\hline & Lémínè-n & ntá & màrè-nè & mùkkú-n & lii-kòotá. \\
\hline & enfant-D & INACP.NEG & éduquer.DETR-GER & & \\
\hline
\end{tabular}

Lenki geden coxo qunbane daqqun kaane!

Creuse le puits d'aujourd'hui en prévision de la soif de demain!

\begin{tabular}{|l|l|l|l|l|l|l|}
\hline & Lènkí & gèdé-n & còxó & qùnbàné & dàqqú-n & kàané ! \\
\hline & aujourd'hui & puits- $\mathrm{D}^{\mathrm{BH}}$ & creuser & demain & soif- $\mathrm{D}^{\mathrm{BH}}$ & devant \\
\hline
\end{tabular}

Lenmen ga n'i ma qasen yari, a wa tin'i haaba d'i qalisin bonondi ya.

Si l'enfant voit sa mère vieille, il dira que son père a gâté son argent.

\begin{tabular}{|c|c|c|c|c|c|c|}
\hline Lénmè-n & gà & n(á) & í & mà & qàsé-n & yàrì, \\
\hline enfant-D & SBD & PROJ & REFL & mère ${ }^{\mathrm{BH}}$ & vieux-D ${ }^{\mathrm{BH}}$ & voir $^{B}$ \\
\hline
\end{tabular}

\begin{tabular}{|l|l|l|l|l|l|l|l|l|}
\hline & à & wá & tì-n(î) & $\hat{\mathbf{i}}$ & hàabá & $\mathbf{d}(\mathbf{a})$ & $\hat{\mathbf{i}}$ & qàlisíín \\
\hline & $3 S \mathrm{SG}$ & INACP & dire-GER & REFL & père $^{\mathrm{BH}}$ & TR & REFL & $\operatorname{argent-D~}^{\mathrm{BH}}$ \\
\hline
\end{tabular}

\begin{tabular}{|l|l|l|}
\hline & bònò-ndí & yà. \\
\hline & s'abîmer-CAUS & FOC \\
\hline
\end{tabular}

Lenmen qenn'i wutaanan ña maxa. 
L'enfant tombe de celui qui le porte.

\begin{tabular}{|l|l|l|l|l|l|l|}
\hline & Lénmè-n & qénn(é) & í & wùt-àaná-n & ñà & màxá. \\
\hline & enfant-D & tomber.GER & REFL & prendre-NMAG-D & FOC & SPHP \\
\hline
\end{tabular}

Max'an du sedi gede di an ga nt'a juhoyen paqqen tu!

Ne te laisse pas tomber dans un puits dont tu ne connais pas la profondeur!

\begin{tabular}{|c|c|c|c|c|c|}
\hline Máx(à) & án & dù & sédi & gèdé & di \\
\hline PRO & $2 S G$ & REFL & jeter & puits & dans \\
\hline
\end{tabular}

\begin{tabular}{|l|l|l|l|l|l|l|l|}
\hline & án & gà & nt(á) & á & jùhòyé-n & pàqqé-n & tù ! \\
\hline & $2 \mathrm{SG}$ & $\mathrm{SBD}$ & $\mathrm{INACP.NEG}$ & $3 \mathrm{SG}^{\mathrm{H}}$ & profondeur- $^{\mathrm{BH}}$ & mesure- $\mathrm{D}^{\mathrm{BH}}$ & connaître.GER $^{\mathrm{B}}$ \\
\hline
\end{tabular}

Maxa an kitten lo yitten do i koromen naxa!

Ne mets pas ta main entre l'arbre et son écorce!

\begin{tabular}{|l|l|l|l|l|l|l|l|l|l|}
\hline & Máxà & án & kìttê-n & ló & yíttè-n & dó & î & kòròmé-n & nàxá ! \\
\hline & PRO & $2 S G$ & main- $\mathrm{D}^{\mathrm{BH}}$ & mettre & arbre-D & avec & REFL & écorce- $\mathrm{D}^{\mathrm{BH}}$ & entre \\
\hline
\end{tabular}

Max'an kittidunñexen yara, t'an taadunñexe!

Ne laisse pas le poisson que tu as dans la main en pensant à celui qui est sous ton pied!

\begin{tabular}{|l|l|l|l|l|}
\hline & Máx(à) & án & kìttì-dù-n-ñèxé-n & yàrá, \\
\hline & PRO & $2 S G$ & main-dans-EP-poisson-D & \\
\hline $\mathrm{BH}$ & laisser \\
\hline
\end{tabular}

\begin{tabular}{|l|l|l|l|}
\hline & $\mathbf{t}(\mathbf{i})$ & án & tàa-dù-n-ñèxê ! \\
\hline & dire & $2 S G$ & pied-dans-EP-poisson. $D^{\mathrm{BH}}$ \\
\hline
\end{tabular}

Maxa baxu jarintesaxunten ya!

'Ne provoque pas le lion qui est couché !'

\begin{tabular}{|l|l|l|l|l|}
\hline & Máxà & bàxú & jàrìntè-sáxú-ntè-n & yá. \\
\hline
\end{tabular}




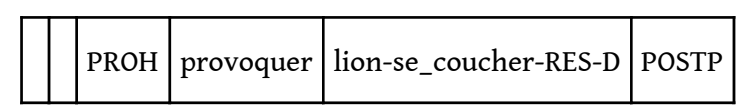

Me ma yigeyen ña na suxuñaaxun lingondini.

C'est de pouvoir manger chacun la mère de l'autre qui fait le charme de la sorcellerie.

\begin{tabular}{|l|l|l|l|l|l|l|}
\hline & Mé & mà & ỳ̀gè-yé-n & nà & nà & súxúñáaxù-n \\
\hline & RECIP & mère $^{\mathrm{BH}}$ & manger-NMLS-D & FOC & INACP & sorcellerie-D \\
\hline
\end{tabular}

\begin{tabular}{|l|l|}
\hline & lingò-ndi-nì. \\
\hline & être-agréable-CAUS-GER \\
\hline
\end{tabular}

Naakaton pilli ya na me hallen tarana.

Ce sont les deux vaches grasses qui se lèchent mutuellement le dos.

\begin{tabular}{|l|l|l|l|l|l|l|l|}
\hline & Nàa-kátò-n & pillì & yá & nà & mé & hàllé-n & tàrà-nà. \\
\hline & vache-gras.PL-D & $\operatorname{deux}^{\mathrm{B}}$ & FOC & INACP & RECIP & dos- $^{\mathrm{BH}}$ & lécher-GER $^{\mathrm{B}}$ \\
\hline
\end{tabular}

Na Alla toxo kanmun di, jarinten lenmen nta sokkinqayen ñigana.

Tant que Dieu sera au ciel, le petit du lion ne mangera pas de fourrage.

\begin{tabular}{|l|l|l|l|l|l|}
\hline & Nà & Allà & tóxó & kànmú-n & dì. \\
\hline & tant_que & Dieu & rester & ciel-D & dans \\
\hline
\end{tabular}

\begin{tabular}{|l|l|l|l|l|l|}
\hline & jàrìnté-n & lènmê-n & ntá & sókkí-n-qàyé-n & ñìà-nà. \\
\hline & lion-D & enfant- $\mathrm{D}^{\mathrm{BH}}$ & INACP.NEG & herbe-EP-frais-D & manger-GER $^{\mathrm{BH}}$ \\
\hline
\end{tabular}

Nallenmun ña nta jarinten qaasaxannen tu, qa naaxooron y'a tu.

Ce sont les veaux qui ne reconnaissent pas le rugissement du lion, mais les vaches adultes le reconnaissent.

\begin{tabular}{|l|l|l|l|l|l|l|}
\hline & Nà-l-lénmù-n & ñá & ntá & jàrìnté-n & qàasà-xànné-n & tù, \\
\hline & vache-EP-enfant.PL-D & FOC & INACP.NEG & lion-D & crier-son-D $\mathrm{D}^{\mathrm{BH}}$ & connaître.GER $^{\mathrm{B}}$ \\
\hline
\end{tabular}
qà nàa-xòorô-n
y(á) à tú. 


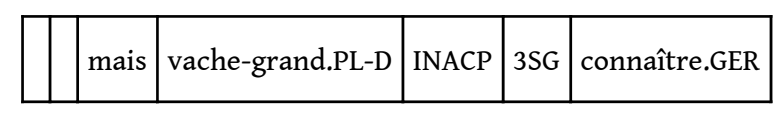

Nan ga na tuuri, a wa tin'i ntaxa hulanqannen mukku.

Quand la vache devient folle, elle dit qu'elle ne comprend plus le peul.

\begin{tabular}{|l|l|l|l|l|l|l|l|}
\hline & Ná-n & gà & ná & tùurì, & à & wá & tì̀-n(í) \\
\hline & vache-D & SBD & PROJ & devenir_fou & 3SG & INACP & dire-GER \\
\hline
\end{tabular}

\begin{tabular}{|l|l|l|l|l|}
\hline & î & ntáxà & húlá-n-qánnè-n & mùkkú. \\
\hline & REFL & CESS & peul-EP-langue-D & entendre.GER \\
\hline
\end{tabular}

«N ñi dagana qatin kin'an ya, q'a joxi killen ya, » ken jaabin ni « yabo » ya yi.

«J'allais te donner du lait, mais il s'est renversé en route », à cela on peut seulement répondre « oui ».

\begin{tabular}{|l|l|l|l|l|l|l|l|}
\hline & « N & ñi & dàgà-ná & qátì-n & kín(i) & án & yà, " \\
\hline & $1 S G$ & PAS & aller-GER & lait-D & donner & 2SG & POSTP \\
\hline
\end{tabular}

\begin{tabular}{|l|l|l|l|l|l|}
\hline & q(à) & à & jòxí & killé-n & yà, " \\
\hline & mais & $3 S G$ & verser.DETR & route-D & POSTP \\
\hline
\end{tabular}

\begin{tabular}{|l|l|l|l|l|l|l|}
\hline & kén & jàabí-n & nì & « yàbó " & yà & yí. \\
\hline & DEM & réponse-D & COPEQ & oui & FOC & POSTP \\
\hline
\end{tabular}

«N ya diinan munda, " hiisire, q' "In ntaxa baqqa misiden noxon di, " janmu ya nt'an kaara.

"J'aime la religion », c'est bien, mais «Je ne sors plus de la mosquée, » c'est que quelque chose ne va pas chez toi.

\begin{tabular}{|c|c|c|c|c|}
\hline$\ll N$ & yá & díinà-n & mùndá, " & hîi-sírè, \\
\hline $1 S G$ & INACP & religion-D & aimer.GER & chose-bon.D \\
\hline
\end{tabular}

\begin{tabular}{|l|l|l|l|l|l|l|l|}
\hline & q(à) & « Ín & ntáxà & báqqá & mìsídè-n & nòxó-n & di, » \\
\hline & mais & $1 S G$ & CESS & sortir.GER & mosquée-D & intérieur-D & dans \\
\hline
\end{tabular}




\begin{tabular}{|l|l|l|l|l|l|}
\hline & jánmù & yá & nt(á) & án & kàará. \\
\hline & paix & FOC & COPLOC.NEG & $2 S G$ & côtée $^{B H}$ \\
\hline
\end{tabular}

Nuxunnen nan ciri yaaxon naxa.

Il convient au nez d'être entre les yeux.

\begin{tabular}{|l|l|l|l|l|l|}
\hline & Núxúnnè-n & nàn & círì & yáaxò-n & nàxá. \\
\hline & nez-D & ST & être_bon & œil.PL-D & entre \\
\hline
\end{tabular}

Qanne ga nta sere be ya, ken la nta yinkolla marana.

Celui qui n'a pas de cou ne peut pas garder une tête.

\begin{tabular}{|l|l|l|l|l|l|l|}
\hline & Qánné & gà & ntá & sèré & bè & yá, \\
\hline & cou & SBD & COPLOC.NEG & personne & REL & POSTP \\
\hline
\end{tabular}

\begin{tabular}{|l|l|l|l|l|l|}
\hline & kén & là & ntá & yínkóllá & màrànà. \\
\hline & DEM & POT & INACP.NEG & tête & garder-GER \\
\hline
\end{tabular}

Qeerin nta tini dangaanan da «N payi!»

Le bonheur ne dit pas au passant « Me voici! »

\begin{tabular}{|l|l|l|l|l|l|l|l|}
\hline & Qéerì-n & ntá & tì-nì & dángàaná-n & dà & « N & páyí ! " \\
\hline & bonheur-D & INACP.NEG & dire-GER & passer-NMAG-D & pour & 1SG & OST \\
\hline
\end{tabular}

Qirisen caxunten ga ke be yalli, leminen ciginten nt'a yalli.

Ce que le vieux voit couché, l'enfant ne le voit pas debout.

\begin{tabular}{|l|l|l|l|l|l|l|}
\hline & Qìrìsé-n & càxù-nté-n & gà & ké & bè & yàllí, \\
\hline & vieux-D & se_coucher-RES-D & & & \\
\hline
\end{tabular}

\begin{tabular}{|l|l|l|l|l|l|}
\hline & lémínè-n & cìgì-ntê-n & nt(á) & à & yàlli. \\
\hline & enfant-D & se_dresser-RES-D & & \\
\hline
\end{tabular}

Qirisen digaamen ga na dalla gunnen di moxo wo moxo, a nta wuyini non ya. 
Si longtemps que la parole du vieux reste en brousse, elle n'y passe pas la nuit.

\begin{tabular}{|l|l|l|l|l|l|l|l|}
\hline & Qìrìsé-n & digàamé-n & gà & ná & dàllà & gùnné-n & dì \\
\hline & vieux-D & parole- $\mathrm{D}^{\mathrm{BH}}$ & $\mathrm{SBD}$ & $\mathrm{PROJ}$ & durer $^{\mathrm{BH}}$ & brousse-D & dans \\
\hline
\end{tabular}

\begin{tabular}{|l|l|l|l|l|l|l|l|l|l|}
\hline & mòxó & wó & mòxò, & à & ntá & wùyì-nì & nón & yà. & \\
\hline & manière & DISTR & manière & B & $3 S G$ & INACP.NEG & passer_la_nuit-GER & là & POSTP \\
\hline
\end{tabular}

Saaraanan ni giden ña yi, an ga na heregeti a yi, an na qenu, a ga na heregeti an ya, an na qenu.

Un parent (père ou mère) est une pierre, si tu le heurtes, tu tombes, s'il te heurte, tu tombes.

\begin{tabular}{|l|l|l|l|l|l|}
\hline & Sàar-áanà-n & ní & gídè-n & ñá & yì, \\
\hline & engendrer-NMAG-D & COPEQ & pierre-D & FOC & POSTP \\
\hline
\end{tabular}

\begin{tabular}{|c|c|c|c|c|c|c|c|c|}
\hline án & gà & ná & hèrègètì & à & yí, & án & ná & qénú, \\
\hline $2 S G$ & SBD & PROJ & heurter $^{B}$ & $3 S G$ & POSTP & $2 S G$ & PROJ & tomber \\
\hline
\end{tabular}

\begin{tabular}{|c|c|c|c|c|c|c|c|c|}
\hline à & gà & ná & hèrègètì & án & yà, & án & ná & qénú. \\
\hline $3 S G$ & SBD & PROJ & heurter ${ }^{B}$ & $2 S G$ & POSTP & $2 S G$ & PROJ & tomb \\
\hline
\end{tabular}

Segaanan qentenmen ga linjo, a tan na battesiren ña yi.

Si celui qui escalade est volubile, c'est qu'il a un bon appui pour son pied.

\begin{tabular}{|l|l|l|l|l|}
\hline & Sèg-áanà-n & qèntènmé-n & gà & línnó, \\
\hline & grimper-NMAG-N & volubilité-D & SBD & être_bon \\
\hline
\end{tabular}

\begin{tabular}{|c|c|c|c|c|c|}
\hline á & tà-n & nà & bátté-sírè-n & ñá & yì. \\
\hline $3 \mathrm{SG}^{\mathrm{H}}$ & pied-D ${ }^{\mathrm{BH}}$ & COPLOC & site-bon-D & FOC & POSTP \\
\hline
\end{tabular}

Sehe yinme da, taro g'a yi!

Parle à une tête qui a des oreilles ! 


\begin{tabular}{|l|l|l|l|l|l|l|l|}
\hline & Sèhé & yínmé & dà, & táró & g(à) & à & yí \\
\hline & parler & tête & pour & oreille & SBD & 3 SG & POSTP \\
\hline
\end{tabular}

Selinyen ga na qur'an kanma, maxa t'a da haasidi, dag'a yinme saxukonpen payi kurukurun noxon di!

Si la poule fait ses besoins sur toi, ne dis pas qu'elle est méchante, va voir l'endroit où elle dort elle-même dans le poulailler !

\begin{tabular}{|l|l|l|l|l|l|l|}
\hline & Sélinyé-n & gà & ná & qùr(ù) & án & kànmá, \\
\hline & poue-D & SBD & PROJ & déféquer & 2SG & sur \\
\hline
\end{tabular}

\begin{tabular}{|l|l|l|l|l|l|l|l|l|}
\hline & máxà & $\mathbf{t}(\mathbf{i})$ & à & dá & háasìdi, & dàg(á) & á & yìnmé \\
\hline & PROH & dire & $3 S G$ & pour & méchant.D & aller & $3 S G^{\mathrm{H}}$ & INT \\
\hline
\end{tabular}

\begin{tabular}{|l|l|l|l|l|l|}
\hline & sàxù-kònpê-n & páyí & kúrúkúrù-n & nòxó-n & di ! \\
\hline & se_coucher-chambre- $\mathrm{D}^{\mathrm{BH}}$ & regarder & poulailler-D & intérieur- $\mathrm{D}^{\mathrm{BH}}$ & dans \\
\hline
\end{tabular}

Sere be nda guranyen lo sanjanceeron tan ya, tenkundagabe ken na a katixannen mukku $y a$.

Celui qui accroche un grelot à la patte du perroquet en entendra le son dans beaucoup de parcelles.

\begin{tabular}{|l|l|l|l|l|l|l|l|l|}
\hline & Sèré & bè & ndá & gùrányè-n & lò & sánjáncéerò-n & tá-n & yà, \\
\hline & personne & REL & SBD.PROJ & grelot-D & mettre & perroquet-D & patte-D & POSTP \\
\hline
\end{tabular}

\begin{tabular}{|l|l|l|l|l|l|l|l|}
\hline & tè-n-kùndà-gábè & kén & nà & á & kàtì-xànné-n & mùkkú & yà. \\
\hline & champ-EP-quartier-beaucoup & DEM & INACP & 3 SGH & frapper-son-D & entendre.GER & FOC \\
\hline
\end{tabular}

Seren d'i haaba taaxu berabaane kanma, ken la nt'an paaba ñaan'an mellenme.

On n'est pas l'égal de son père parce qu'on s'asseoir à la même place.

\begin{tabular}{|l|l|l|l|l|l|l|l|}
\hline & Sèré-n & $\mathbf{d}(\mathbf{o})$ & í & hàabá & táaxú & bérá-báané & kànmá, \\
\hline & personne-D & avec & REFL & père ${ }^{\mathrm{BH}}$ & s'asseoir & place-un & sur \\
\hline
\end{tabular}

\begin{tabular}{|l|l|l|l|l|l|l|l|l|}
\hline & kén & là & nt(á) & án & pàabá & ǹàa-n(à) & án & mèllènmê. \\
\hline
\end{tabular}




\begin{tabular}{|c|c|c|c|c|c|c|c|}
\hline DEM & POT & INACP & $2 S G$ & père ${ }^{B H}$ & faire-GER ${ }^{B}$ & $2 S G$ & égal. $\mathrm{D}^{\mathrm{BH}}$ \\
\hline
\end{tabular}

Seren ga na muxu moxo wo moxo, an ñaaxen y'an du yi.

On a beau se cacher, on reste à la vue de ses propres yeux.

\begin{tabular}{|l|l|l|l|l|l|l|l|}
\hline & Sèré-n & gà & ná & mùxù & mòxó & wó & mòxò, \\
\hline & personne-D & SBD & PROJ & se_cacher & manière & DISTR & manière \\
\hline
\end{tabular}

\begin{tabular}{|l|l|l|l|l|l|l|}
\hline & án & ñàaxê-n & $\mathbf{y}(\mathbf{a})$ & án & dù & yí. \\
\hline & $2 S G$ & œil- $\mathrm{D}^{\mathrm{BH}}$ & COPLOC & $2 \mathrm{SG}$ & REFL $^{\mathrm{BH}}$ & POSTP \\
\hline
\end{tabular}

Sere n'maxa i sin katu i yinmen ya, ti siyanyen taax'a yi.

On ne frappe pas son cheval à la tête parce qu'une mouche s'est posée dessus.

\begin{tabular}{|l|l|l|l|l|l|l|l|l|l|}
\hline & Sèré & n(àn) & máxà & î & sì-n & kátú & í & yìnmé-n & yà \\
\hline & personne & SUBJ & PROH & REFL & cheval-D & frapper & REFL & tête-D ${ }^{\mathrm{BH}}$ & POSTP \\
\hline
\end{tabular}

\begin{tabular}{|l|l|l|l|l|l|}
\hline & tî́ & síyányè-n & táax(ú) & à & yí. \\
\hline & dire & mouche-D & se_poser & $3 S G$ & POSTP \\
\hline
\end{tabular}

Seren nt'i yaaxen loxono i qanan ya, an ñinme nan payindi t'an kohen ya.

On ne prête pas ses yeux à son ami pour regarder avec sa nuque.

\begin{tabular}{|l|l|l|l|l|l|l|l|l|}
\hline & Sèrê-n & nt(á) & $\hat{\mathbf{i}}$ & yàaxé-n & lòxò-nò & $\hat{\mathbf{i}}$ & qàná-n & yà, \\
\hline & persone-D & INACP & REFL & œil- $\mathrm{D}^{\mathrm{BH}}$ & prêter-GER & $\mathrm{REFL}$ & ami-D $^{\mathrm{BH}}$ & POSTP \\
\hline
\end{tabular}

\begin{tabular}{|l|l|l|l|l|l|l|l|l|}
\hline & án & ñìnmé & nàn & páyí-ndi & $\mathbf{t}(\mathbf{i})$ & án & kòhé-n & yà. \\
\hline & $2 S G$ & INT & SUBJ & regarder-ANTIP & avec & $2 S G$ & nuque-D & POSTP \\
\hline
\end{tabular}

Seren ya gullan giriran tu, q'an nt'a qenura tu.

On sait d'où part une pierre, mais pas où elle va tomber. 


\begin{tabular}{|l|l|l|l|l|l|}
\hline & Sèrê-n & yá & gúllà-n & gìrì-rá-n & tù, \\
\hline & personne-D & INACP & pierre-D & partir-lieu-D & Connaître.GER \\
\hline
\end{tabular}

\begin{tabular}{|l|l|l|l|l|l|l|}
\hline & q(à) & án & $\operatorname{nt}(\mathbf{a})$ & á & qènù-rá & tù. \\
\hline & mais & $2 S G$ & INACP.NEG & $3 S G^{\mathrm{H}}$ & tomber-lieu $^{\mathrm{BH}}$ & connaître.GER $^{\mathrm{B}}$ \\
\hline
\end{tabular}

Sereyillanten ña na dinmen timi lungunten da.

C'est l'idiot qui bat le tambour pour un sourd.

\begin{tabular}{|l|l|l|l|l|l|}
\hline & Sèrè-yíllà-nté-n & ñà & nà & dìnmé-n & tìnmì \\
\hline & personne-retourner-RES-D & FOC & INACP & tambour-D & battre.GER $^{B}$ \\
\hline
\end{tabular}

\begin{tabular}{|l|l|l|}
\hline & lùngù-nté-n & dà. \\
\hline & devenir_sourd-RES-D & pour \\
\hline
\end{tabular}

Si be g'an qenundini, an nt'a nuxunnen yalli.

Le cheval qui te fera tomber, tu ne vois pas son nez.

\begin{tabular}{|l|l|l|l|l|l|}
\hline & Sí & bè & g(á) & án & qénú-ndí-ní, \\
\hline & cheval & REL & SBD & $2 S G$ & tomber-CAUS-GER \\
\hline
\end{tabular}

\begin{tabular}{|l|l|l|l|l|l|}
\hline & án & nt(á) & á & nùxùnné-n & yàlli. \\
\hline & $2 S G$ & INACP.NEG & $3 S^{\mathrm{H}}$ & nez-D & \\
\hline
\end{tabular}

Singiyen nan lingi ñogomen da, q'a hallen nta dunyen'a da.

Le dromadaire aimerait s'allonger sur le dos, mais son dos ne lui permet pas.

\begin{tabular}{|l|l|l|l|l|l|}
\hline & Sîngì-yé-n & nàn & língí & ñogòmé-n & dà, \\
\hline & se_coucher_sur_le_dos-NMLS-D & ST & être_agréable & dromadaire-D & pour \\
\hline
\end{tabular}

\begin{tabular}{|l|l|l|l|l|l|l|l|}
\hline & q(à) & á & hàllê-n & ntá & dùnyèn(è) & à & dá. \\
\hline & mais & $3 S^{\mathrm{H}} \mathrm{H}^{\mathrm{H}}$ & dos-D $^{\mathrm{BH}}$ & INACP.NEG & accepter-GER & $3 \mathrm{SG}$ & pour \\
\hline
\end{tabular}

Sokke be ga seren ñaaxen karana, an nta ken yalli. 
On ne voit pas le brin d'herbe qui va vous crever l'œil.

\begin{tabular}{|l|l|l|l|l|l|l|}
\hline & Sókké & bè & gá & sèré-n & ñàaê-n & kárá-ná, \\
\hline & herbe & REL & SBD.INACP & personne-D & œil-D $\mathrm{D}^{\mathrm{BH}}$ & casser-GER \\
\hline
\end{tabular}

\begin{tabular}{|l|l|l|l|l|}
\hline & án & ntá & kén & yàlli. \\
\hline & 2SG & INACP.NEG & DEM & voir.GER \\
\hline
\end{tabular}

Sokken do yinben la nta sangana doome.

La paille et le feu ne peuvent pas jouer ensemble.

\begin{tabular}{|l|l|l|l|l|l|l|l|}
\hline & Sókkè-n & dó & yìnbé-n & là & ntá & sàngà-nà & dóòmé. \\
\hline & paille-D & avec & feu-D & POT & INACP.NEG & jouer-GER & ensemble \\
\hline
\end{tabular}

Suxuñan ña mungunu, q'a ga da ke be renmen ñiga, ken nta mungunu.

C'est le sorcier qui oublie, mais celui dont il a mangé l'enfant n'oublie pas.

\begin{tabular}{|l|l|l|l|l|l|l|l|l|l|}
\hline & Súxúñà-n & ñá & mùngù-nù, & q(à) & à & gà & dà & ké & bè \\
\hline & sorcier-D & FOC & oublier-GER & mais & $3 S G$ & $S B D$ & $T R$ & $D E M$ & $R E L$ \\
\hline
\end{tabular}

\begin{tabular}{|l|l|l|l|l|l|}
\hline & rènmê-n & ñígá, & kên & ntá & mùngù-nù. \\
\hline & enfant- $\mathrm{D}^{\mathrm{BH}}$ & manger & DEM & INACP.NEG & oublier-GER \\
\hline
\end{tabular}

Tan ya yuñen di, samaqqen ya yuñen di, kootabaane m'i ga da me ñi.

Le pied est sur le sol, le serpent est sur le sol, un jour il faudra qu'ils se rencontrent.

\begin{tabular}{|l|l|l|l|l|l|l|l|l|}
\hline & Tâ-n & yá & yùñé-n & dì, & sámáqqè-n & yá & yùñé-n & dì, \\
\hline & pied & COPLOC & sol-D & dans & serpent-D & COPLOC & sol-D & dans \\
\hline
\end{tabular}

\begin{tabular}{|l|l|l|l|l|l|l|l|}
\hline & kòotà-báané & $\mathbf{m}(\mathbf{a})$ & $\mathbf{i}$ & gà & dà & mé & ñi. \\
\hline & jour-un & OBLG & $3 \mathrm{PL}$ & $\mathrm{SBD}$ & $\mathrm{TR}$ & $\mathrm{RECIP}$ & trouver \\
\hline
\end{tabular}

Tinkanbon pilli ga hungu me yi, hunbuunen ña na baanen ya. 
Si l'une des deux fesses dépasse l'autre, c'est qu'elle a un abcès.

\begin{tabular}{|l|l|l|l|l|l|l|}
\hline & Tínkánbò-n & pìlli & gà & húnyù & mé & ỳ̀, \\
\hline & fesse.PL-D & deux $^{\mathrm{B}}$ & $\mathrm{SBD}$ & être_supérieur & RECIP & POSTP \\
\hline
\end{tabular}

\begin{tabular}{|l|l|l|l|l|l|}
\hline & húnbùuné-n & ñà & nà & báanè-n & yá. \\
\hline & abcès-D & FOC & COPLOC & un-D & POSTP \\
\hline
\end{tabular}

Tonyun la wa dallana sellan ya, q'a nta wuyini no.

La vérité peut rester longtemps dehors, mais elle n'y passe pas la nuit.

\begin{tabular}{|l|l|l|l|l|l|l|}
\hline & Tònyú-n & là & wá & dàllà-ná & séllà-n & yá, \\
\hline & vérité-D & POT & INACP & durer-GER & dehors-D & POSTP \\
\hline
\end{tabular}

\begin{tabular}{|l|l|l|l|l|l|}
\hline & q(à) & à & ntá & wùyì-nì & nó. \\
\hline & mais & $3 S G$ & INACP.NEG & passer_la_nuit-GER & là \\
\hline
\end{tabular}

Tunguron ga qenu wo qenu, a do yuñen la nta ñerono.

La termitière a beau s'effondrer, elle ne peut pas tomber au niveau du sol.

\begin{tabular}{|l|l|l|l|l|l|}
\hline & Túngúrò-n & gà & qénú & wó & qènù, \\
\hline & termitière-D & SBD & tomber & CONC & tomber $^{B}$ \\
\hline
\end{tabular}

\begin{tabular}{|l|l|l|l|l|l|l|}
\hline & á & dò & yùñé-n & là & ntá & ñèrò-nò. \\
\hline & $3 S G^{\mathrm{H}}$ & avec & sol-D & POT & INACP.NEG & devenir_égal-GER \\
\hline
\end{tabular}

Wurudunnan cu binnen ña ni.

La nuit toutes les vaches sont noires.

\begin{tabular}{|c|c|c|c|c|}
\hline Wùrù-dù-n-nâ-n & cú & bìnné-n & ñà & ní. \\
\hline nuit-dans-EP-vache-D & tous & noir- $\mathrm{D}^{\mathrm{BH}}$ & FOC & COPEQ \\
\hline
\end{tabular}

Yaagun ga riini, an ñi ñonqolono misidiberan ya. 
Quand la honte est arrivée, tu étais accroupi dans la cour de la mosquée.

\begin{tabular}{|l|l|l|l|l|l|l|}
\hline & Yàagú-n & gà & ríi-ní, & án & ñi & ñónqólò-nó \\
\hline & honte-D & SBD.COPLOC & venir-GER & 2SG & PAS & s'accroupir-GER \\
\hline
\end{tabular}

\begin{tabular}{|l|l|l|}
\hline & mìsìi-bérà-n & yá. \\
\hline & mosquée-cour-D & POSTP \\
\hline
\end{tabular}

Yaaxo beenu ga ma texe doome, i ra nta yuñini doome.

Des yeux qui ne se sont pas fermés ensemble ne peuvent pas s'ouvrir ensemble.

\begin{tabular}{|l|l|l|l|l|l|l|}
\hline & Yáaxó & bèe-nú & gà & má & tèxè & dóomè, \\
\hline & œil.PL & REL-PL & SBD & ACP.NEG & se_fermer & ensemble \\
\hline
\end{tabular}

\begin{tabular}{|l|l|l|l|l|l|}
\hline & ì & rà & ntá & yùñì-nì & dóomè. \\
\hline & $3 \mathrm{PL}$ & POT & INACP.NEG & s'ouvrir-GER & ensemble \\
\hline
\end{tabular}

Yaxarixorotinten nt'i kiina bannan yalli.

La femme trop pressée ne verra pas son mari riche.

\begin{tabular}{|l|l|l|l|l|l|l|}
\hline & Yàxàrì-xórótí-ntè-n & nt(á) & $\hat{\mathbf{i}}$ & kìiná & bànná-n & yàlli. \\
\hline & femme-se_dépêcher-RES-D & INACP.NEG & REFL & mari & & riche-D \\
\hline
\end{tabular}

Yelingen do dongo hilli nta kanpini.

L'oiseau ne peut pas voler avec deux jabots.

\begin{tabular}{|l|l|l|l|l|l|l|}
\hline & Yèlínyè-n & dó & dóngò & hìllì & ntá & kànpì-nì. \\
\hline & oiseau-D & avec & jabot.PL & deux $^{\mathrm{B}}$ & INACP.NEG & voler-GER \\
\hline
\end{tabular}




\section{NOTES}

1. La forme d'accompli du verbe 'ṹî́ '(se) trouver', combinée au gérondif d'un autre verbe, fonctionne comme auxiliaire à valeur de passé.

2. La particule intensive yìnmé résulte de la grammaticalisation de yínmè 'tête', le ton $\mathrm{BH}$ s'expliquant étymologiquement par le morphotonème qui marque les noms en fonction de tête d'une construction génitivale. Le marqueur de détermination permet de faire la distinction entre par exemple ń ñinmé (non déterminé) 'moi-même' et ń ñinmê (déterminé) 'ma tête'.

3. Pour 'Le jour s'est levé', le soninké utilise une expression (Yíllèn káré) dans laquelle le nom sujet et yíllè, du verbe yillà 'retourner, transformer', à comprendre ici comme se référant au cycle du jour et de la nuit. Quand au verbe káré, forme détransitivisée de kárá 'casser', son sens premier est 'se casser', et pour expliquer son emploi ici, mon consultant suggère une métaphore qui assimile le lever du jour à une éclosion (car parmi ses emplois possibles, káré peut être l'équivalent du français 'éclore').

4. Tunkara, Buubu Yaamadu et Mahanmadu Bonmu (2012). Taalinkitabe (Proverbes en langue soninké), 3ème édition. SIL Mali.

5. ñá est le verbe 'faire', utilisé intransitivement au sens de 'devenir', 'être'.

6. 'tí 'que' est issu de 'tí 'dire' par grammaticalisation.

\section{AUTEUR}

DENIS CREISSELS

Université Lyon 2

denis.creissels@univ-lyon2.fr

http://deniscreissels.fr 